Supporting Information

\title{
Poly(benzyl ether) Dendrimers Functionalized at the Core with Palladium Bis(N-Heterocyclic Carbene) Complexes as Catalysts for the Heck Coupling Reaction
}

Alba M. Ortiz, ${ }^{\dagger}$ Alberto Sánchez-Méndez, ${ }^{\dagger}$ Ernesto de Jesús, ${ }^{*, \dagger}$ Juan C. Flores, ${ }^{*}{ }^{\dagger \dagger}$ Pilar Gómez-Sal ${ }^{\dagger}$ and Francisco Mendicuti ${ }^{\ddagger}$

†Departamento de Química Orgánica y Química Inorgánica, Universidad de Alcalá, Edificio de Farmacia, Campus Universitario, E28871 Alcalá de Henares, Madrid, Spain

*Departamento de Química Analítica, Química Física e Ingeniería Química, Universidad de Alcalá E-mail: ernesto.dejesus@uah.es; juanc.flores@uah.es; Fax: +34 918854683 


\section{Table of contents}

1. General procedures and materials

2. Preparation and characterization data for imidazolium bromides $\mathbf{1 a}-\mathbf{4 a}$ and $\mathbf{1 b}-\mathbf{4 b} \ldots \ldots \ldots . . . \quad \mathrm{S} 4$

3. Preparation and characterization data for NHC-Ag complexes $5 \mathbf{a}-\mathbf{8 a}$ and $\mathbf{5 b}-\mathbf{8 b} \quad \ldots \ldots \ldots \ldots . . . . . \quad \mathrm{S} 8$

4. Preparation and characterization data for NHC-Pd complexes $\mathbf{9 a}-\mathbf{1 2 a}$ and $\mathbf{9 b}-\mathbf{1 2 b} \ldots \ldots \ldots . . . \quad \mathrm{S} 12$

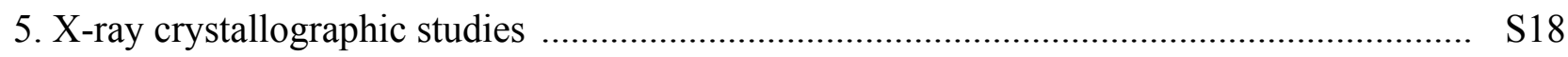

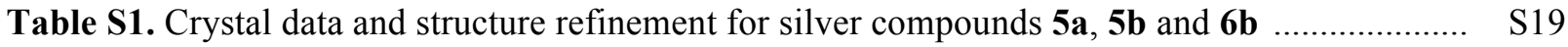

Table S2. Crystal data and structure refinement for palladium compounds

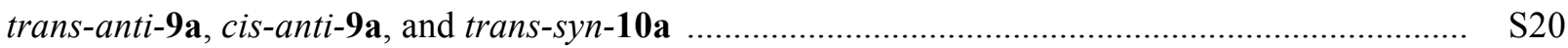

Figure S1. Molecular structure found for cis-anti-9a ............................................................ S2 1

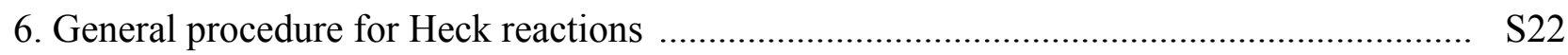

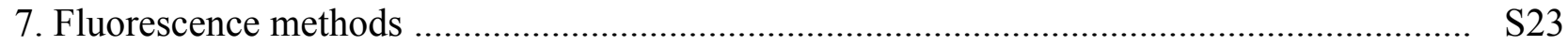

Figure S2. Absorption spectra for 9a-12a dendrimer series in $\mathrm{CH}_{2} \mathrm{Cl}_{2}$ at $25{ }^{\circ} \mathrm{C}$. Superimposed the 280-480 nm absorption region is enlarged. Spectrum for $\mathrm{PdBr}_{2}(\mathrm{cod})$ is included. .................... S25

Table S3. Some photophysical parameters for $\mathrm{G} n(\mathbf{9 a - 1 2 a})$ dendrimers in $\mathrm{CH}_{2} \mathrm{Cl}_{2}$ at $25{ }^{\circ} \mathrm{C}$......... S25

Figure S3. Stern-Volmer plots of the fluoresce intensity measured at the max. of emission band $(420 \mathrm{~nm})$ from the Pd-core for dilute solutions of 9a-12a dendrimers in $\mathrm{CH}_{2} \mathrm{Cl}_{2}$ at $25{ }^{\circ} \mathrm{C}$.

The quencher was diacetyl. Symbols are: 9a $(\Delta) ; 10 a(\bullet) ; 11 a(\square)$ and 12a $(\nabla)$.

Table S4. Quenching parameters obtained from the Stern-Volmer plot depicted in Figure S3 for $9 \mathrm{a}-10 \mathrm{a} / \mathrm{CH}_{2} \mathrm{Cl}_{2}$ dilute solutions at $25^{\circ} \mathrm{C}$ by using as quencher diacetyl.

Figure S4. Coordinate system used for $9 \mathbf{a}-12 \mathrm{a}$ dendrimers in the theoretical MD simulations, exemplified for 10a.

Figure S5. (Upper panel) Hydrodynamics volumes for 9a-12a in DMF (অ), acetone (•) (both from DOSY NMR experiments) obtained at $25^{\circ} \mathrm{C}$. Van der Waals $(\nabla)$ and total volumes $(\square)$ obtained from van der Waals radii and from the values of the average of the root-mean-square radius of gyration respectively. The latter obtained from the analysis of the $5 \mathrm{~ns}$ MD trajectories at $300 \mathrm{~K}$ under the assumption of a 2:1 mixture of trans-syn and trans-anti rotamers. (Bottom panel) experimental and theoretical dendrimer densities obtained from the volumes of the upper panel.

Figure S6. Density of the centers of mass of the phenyl ring from dendrons and mesityl groups (number of centers per $\AA^{3}$ ) from the analysis of the $5 \mathrm{~ns}$ MD trajectories at $300 \mathrm{~K}$ for the trans-syn and trans-anti rotamers of the four dendrimer generations (G0-G4) as a function of the $r$ polar coordinate, the Pd-core being located at the center of a coordinate system (see Figure S4). 


\section{General procedures and materials}

All operations were performed under an argon atmosphere using Schlenk or dry-box techniques. 1-Mesityl- and 1-(2,6-diisopropylphenyl)-imidazole, ${ }^{\mathrm{S} 1}\left[\mathrm{PdX}_{2}(\mathrm{COD})\right](\mathrm{X}=\mathrm{Cl}, \mathrm{Br}),{ }^{\mathrm{S} 2}$ and dendritic benzyl bromides $(\mathrm{G} n$-dend $) \mathrm{Br}(\mathrm{G} 1-\mathrm{G} 3)^{\mathrm{S} 3}$ were prepared according to literature procedures. Unless otherwise stated, reagents were obtained from commercial sources and used as received. Solvents were dried and distilled prior to use under argon as described elsewhere. ${ }^{\mathrm{S} 4}$ Alternatively, solvents of reagent grade (Baker and SDS) were purified by flash column chromatography and collected under urgon, using a MBraun MB SPS solvent purification device. NMR spectra were recorded at room temperature using Varian Mercury 300, Unity 300, or Unity 500 Plus spectrometers. Chemical shifts $\left(\delta\right.$, parts per million) are quoted relative to $\mathrm{SiMe}_{4}\left({ }^{1} \mathrm{H},{ }^{13} \mathrm{C}\right)$ and $\mathrm{MeNO}_{2}\left({ }^{15} \mathrm{~N}\right)$, and were measured by internal referencing to the ${ }^{13} \mathrm{C}$ and residual ${ }^{1} \mathrm{H}$ resonances of the deuterated solvents, or by the substitution method in the case of ${ }^{15} \mathrm{~N}$. When required, NOESY, TOCSY and twodimensional HSQC and HMBC experiments, were performed for unequivocal assignment of resonances. Coupling constants $(J)$ are given in Hz. The following abbreviations/notations are used: Mes refers to the mesityl group, ${ }^{i} \mathrm{Pr}_{2} \mathrm{Ph}$ to 2,6-diisopropylphenyl, Imz to the imidazole ring, $\mathrm{Ph}$ to aromatic ring of terminal benzyl groups, Ar to internal rings of benzyl ethers, and ipso refers to the first ring-position on going from the azole ligand. The diffusion coefficients were measured at 25 ${ }^{\circ} \mathrm{C}$ from solutions of $1 \mathrm{mg}$ of the complex under study in $0.75 \mathrm{~mL}$ of solvent $\left(\mathrm{DMF}-\mathrm{d}_{7}\right.$ or acetone$\mathrm{d}_{6}$ ), by using the Dbppste (DOSY Bipolar Pulse Simulated Echo) pulse sequence in a Varian NMR System 500 equipped with a high accuracy variable temperature unit $\left( \pm 0.1^{\circ} \mathrm{C}\right)$, a Performa IV PFG amplifier, and a Z-PFG Triple Resonance 5-mm probe. Fine calibration of the PFG strength (DAC_to_G unit) was performed with an $\mathrm{H}_{2} \mathrm{O} / \mathrm{HDO}(2 \mathrm{~Hz})$ sample as standard supplied by Varian $\left(D=19.04 \times 10^{-10} \mathrm{~m}^{2} / \mathrm{s}\right.$ at $\left.25^{\circ} \mathrm{C}\right)$. The diffusion NMR data were acquired over 64 scans, with settings pw90, an acquisition time of $3 \mathrm{~s}$, a relaxation delay of $2 \mathrm{~s}$, in each one of the 15 steps of the gradient level array between 1 and $50 \mathrm{G} / \mathrm{cm}(50 \mathrm{~ms}$ of diffusion delay and $2 \mathrm{~ms}$ of total defocusing time). The experimental data $(32 \mathrm{~K} \times 1 \mathrm{~K})$ was treated with the "DOSY" software from VNMRJ2.1B. IR spectra were recorded on a Perkin-Elmer FT-IR Spectrum-2000 spectrophotometer. The Analytical Services of the Universidad de Alcalá performed the C, H, and $\mathrm{N}$ analyses using a LECO CHNS-932 microanalyzer, and recorded the mass spectra using Agilent G3250AA LC/MSD TOF Multi (ESI) or Agilent 6210-TOF LC/MS (Ion source ESI Dual) mass spectrometers. The progress of the Heck reactions was determined with a HP-5890 Series II gas chromatograph equipped with a FID detector, using an Agilent DB-1 capillary column (15 m, 0.25 $\mathrm{mm}$ i.d., $0.10 \mu \mathrm{m} \mathrm{df}$ ) under the following conditions: injector and detector temperatures: 250 and $260{ }^{\circ} \mathrm{C}$, respectively; oven temperature program: isotherm at $120^{\circ} \mathrm{C}$. 


\section{Preparation and characterization data for imidazolium bromides $1 \mathrm{a}-4 \mathrm{a}$ and $\mathbf{1 b}-\mathbf{4 b}$}

The corresponding benzyl wedge $(\mathrm{G} n-d e n d) \mathrm{Br}(n=0,1,2,3)$ and [1-(aryl)Imz] $(\operatorname{aryl}=$ Mes or $\left.{ }^{i} \mathrm{Pr}_{2} \mathrm{Ph}\right)$ were combined in an ampoule equipped with a PTFE valve together with acetone (20-25 $\mathrm{mL})$. The tube was sealed and the mixture stirred at $60{ }^{\circ} \mathrm{C}$ until completion of the reaction (4-6 h, overnight, $24 \mathrm{~h}$, and 2 days, for $\mathbf{1}, \mathbf{2}, \mathbf{3}$, and 4 bromides, respectively). Upon cooling of resulting solution at $0{ }^{\circ} \mathrm{C}$ (1a) and addition of cold pentane (1) $)$, or removal of the solvent followed by stirring in diethyl ether for a few hours $(\mathbf{2 a}-\mathbf{4 a})$ or by several washings with cold diethyl ether $(2 \times$ $15 \mathrm{~mL}, \mathbf{2 b}-\mathbf{4 b}$ ), resulted in the precipitation of the title compounds as white solids (beige in the case of $3 \mathbf{b}$ and $\mathbf{4 b}$ ) that can be washed with pentane.

[1-Mes-3-(G0-dend) Imz] ${ }^{+} \mathbf{B r}^{-}$(1a): $\mathrm{PhCH}_{2} \mathrm{Br}(0.35 \mathrm{~mL}, 2.9 \mathrm{mmol})$ and 1-(Mes) $\mathrm{Imz}(500 \mathrm{mg}$, $2.68 \mathrm{mmol})$. Yield: $820 \mathrm{mg}, 86 \% .{ }^{1} \mathrm{H}$ NMR $\left(\mathrm{CDCl}_{3}\right): \delta=10.53\left(\mathrm{~s}, 1 \mathrm{H}, \mathrm{Imz}-\mathrm{H}^{2}\right), 7.72\left(\mathrm{t}, J_{\mathrm{H}, \mathrm{H}}=1.8\right.$, $\left.1 \mathrm{H}, \mathrm{Imz}-\mathrm{H}^{5}\right), 7.60-7.56(\mathrm{~m}, 2 \mathrm{H}, m-\mathrm{Ph}), 7.35-7.32(\mathrm{~m}, 3 \mathrm{H}, o-$ and $p-\mathrm{Ph}), 7.11\left(\mathrm{t}, J_{\mathrm{H}, \mathrm{H}}=1.8,1 \mathrm{H}, \mathrm{Imz}-\right.$ $\mathrm{H}^{4}$ ), 6.93 (s, 2H, m-Mes), 5.92 (s, 2H, $\mathrm{CH}_{2}$ ), 2.28 (s, 3H, Mes- $\left.p-M e\right), 2.00$ ppm (s, 6H, Mes-o-Me). ${ }^{13} \mathrm{C}\left\{{ }^{1} \mathrm{H}\right\} \mathrm{NMR}\left(\mathrm{CDCl}_{3}\right): \delta=141.1$ (Imz-C $\left.{ }^{2}\right), 137.6$ (p-Mes), 134.0 (o-Mes), 133.5 (ipso-Ph), 130.6 (ipso-Mes), 129.7 and $129.3\left(o-\right.$ and $m$-Ph), 129.2 ( $m$-Mes), $129.0(p-\mathrm{Ph}), 123.2\left(\mathrm{Imz}^{\left.-\mathrm{C}^{5}\right), 122.9}\right.$ $\left(\mathrm{Imz}-\mathrm{C}^{4}\right), 53.2\left(\mathrm{CH}_{2}\right), 21.0(\mathrm{Mes}-p-M e), 17.6 \mathrm{ppm}(\mathrm{Mes}-o-M e)$. IR (KBr pellet): $v=1606(\mathrm{~m})$ and 1456 (s, C-C $\mathrm{C}_{\text {arom }}$ ), $1496 \mathrm{~cm}^{-1}$ (s, CNC). Elemental analysis (\%) calcd for $\mathrm{C}_{19} \mathrm{H}_{21} \mathrm{BrN}_{2}$ (357.29): $\mathrm{C}$ 63.87, $\mathrm{H}$ 5.92, $\mathrm{N}$ 7.84; found: $\mathrm{C}$ 63.99, $\mathrm{H}$ 5.86, $\mathrm{N}$ 7.92. MS $\left(\mathrm{ESI}^{+}-\mathrm{TOF}\right.$ in $\left.\mathrm{CH}_{2} \mathrm{Cl}_{2} / \mathrm{MeOH} / \mathrm{NH}_{4} \mathrm{HCOO} 5 \mathrm{mM}\right): m / z=277.17[\mathrm{M}-\mathrm{Br}]^{+}$.

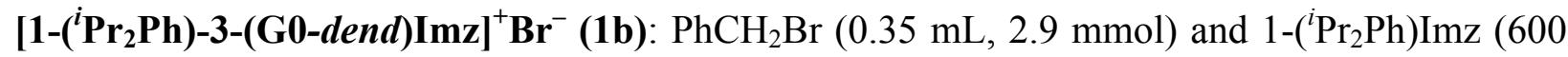
mg, $2.63 \mathrm{mmol})$. Yield: $838 \mathrm{mg}, 80 \% .{ }^{1} \mathrm{H}$ NMR $\left(\mathrm{CDCl}_{3}\right): \delta=10.66\left(\right.$ broad s, $\left.1 \mathrm{H}, \mathrm{Imz}-\mathrm{H}^{2}\right), 7.64(\mathrm{t}$, $\left.J_{\mathrm{H}, \mathrm{H}}=1.8,1 \mathrm{H}, \mathrm{Imz}_{-} \mathrm{H}^{5}\right), 7.57-7.60(\mathrm{~m}, 2 \mathrm{H}, m-\mathrm{Ph}), 7.51\left(\mathrm{t},{ }^{3} J_{\mathrm{H}, \mathrm{H}}=7.8,1 \mathrm{H}, p-{ }^{i} \mathrm{Pr}{ }_{2} \mathrm{Ph}\right), 7.38-7.41(\mathrm{~m}$, $3 \mathrm{H}, o$ - and $p-\mathrm{Ph}), 7.28\left(\mathrm{~d},{ }^{3} J_{\mathrm{H}, \mathrm{H}}=7.8,2 \mathrm{H}, m^{i}{ }^{i} \mathrm{Pr}_{2} \mathrm{Ph}\right), 7.09\left(\mathrm{t}, J_{\mathrm{H}, \mathrm{H}}=1.8,1 \mathrm{H}, \mathrm{Imz}_{-} \mathrm{H}^{4}\right), 6.06(\mathrm{~s}, 2 \mathrm{H}$, $\left.\mathrm{CH}_{2}\right), 2.24\left(\mathrm{sep},{ }^{3} J_{\mathrm{H}, \mathrm{H}}=7.8,2 \mathrm{H}, \mathrm{CHMe}\right), 1.21\left(\mathrm{~d},{ }^{3} J_{\mathrm{H}, \mathrm{H}}=7.8,6 \mathrm{H}, \mathrm{CHMe} e_{2}\right), 1.11\left(\mathrm{~d},{ }^{3} J_{\mathrm{H}, \mathrm{H}}=7.8,6 \mathrm{H}\right.$, $\mathrm{CHMe} 2) .{ }^{13} \mathrm{C}\left\{{ }^{1} \mathrm{H}\right\}$ NMR $\left(\mathrm{CDCl}_{3}\right): \delta=145.2\left(p-{ }^{i} \mathrm{Pr}_{2} \mathrm{Ph}\right), 138.0\left(\mathrm{Imz} \mathrm{C}^{2}\right), 133.7$ (ipso-Ph), 131.8 (o$\left.{ }^{i} \mathrm{Pr}_{2} \mathrm{Ph}\right), 130.1$ (ipso- $\left.{ }^{i} \mathrm{Pr}_{2} \mathrm{Ph}\right), 129.1$ and 129.3 (o-, $m$ - and $p$-Ph overlapping), $124.6\left(m-{ }^{i} \mathrm{Pr}_{2} \mathrm{Ph}\right), 124.0$ $\left(\mathrm{Imz}-\mathrm{C}^{5}\right), 123.1\left(\mathrm{Imz}^{4} \mathrm{C}^{4}\right), 53.2\left(\mathrm{CH}_{2}\right), 28.6\left(\mathrm{CHMe}_{2}\right), 24.4\left(\mathrm{CHMe}_{2}\right), 23.9\left(\mathrm{CHMe}_{2}\right)$. IR $(\mathrm{KBr}$ pellet): $v=1601(\mathrm{~m}), 1555$ and $1460\left(\mathrm{~s}, \mathrm{C}-\mathrm{C}_{\mathrm{arom}}\right), 1496 \mathrm{~cm}^{-1}$ (s, CNC). Elemental analysis (\%) calcd for $\mathrm{C}_{22} \mathrm{H}_{27} \mathrm{BrN}_{2}$ (399.37): C 66.16, H 6.81, N 7.01; found: C 65.91, H 6.75, N 6.91. MS (ESI TOF in $\left.\mathrm{CH}_{2} \mathrm{Cl}_{2} / \mathrm{MeOH} / \mathrm{NH}_{4} \mathrm{HCOO} 5 \mathrm{~mm}\right): m / z=319.22[\mathrm{M}-\mathrm{Br}]^{+}$.

[1-Mes-3-(G1-dend)Imz] ${ }^{+} \mathbf{B r}^{-}$(2a): (G1-dend) $\mathrm{Br}$ (735 mg, $\left.1.92 \mathrm{mmol}\right)$ and 1-(Mes)Imz (372 mg, $2.00 \mathrm{mmol})$. Yield: $880 \mathrm{mg}, 80 \%$. ${ }^{1} \mathrm{H}$ NMR $\left(\mathrm{CDCl}_{3}\right): \delta=10.65$ (s, 1H, Imz-H $\left.{ }^{2}\right), 7.41-7.28(\mathrm{~m}$, $11 \mathrm{H}, \mathrm{Ph}$ and Imz-H $\left.\mathrm{H}^{5}\right), 7.02\left(\mathrm{~s}, 1 \mathrm{H}, \mathrm{Imz}-\mathrm{H}^{4}\right), 6.97(\mathrm{~s}, 2 \mathrm{H}, m-\mathrm{Mes}), 6.82\left(\mathrm{~d},{ }^{4} J_{\mathrm{H}, \mathrm{H}}=2.2,2 \mathrm{H}, o-\mathrm{Ar}\right)$, 
$6.61\left(\mathrm{t},{ }^{4} J_{\mathrm{H}, \mathrm{H}}=2.2,1 \mathrm{H}, p-\mathrm{Ar}\right), 5.85\left(\mathrm{~s}, 2 \mathrm{H}, \mathrm{CH}_{2}\right), 5.04\left(\mathrm{~s}, 4 \mathrm{H}, \mathrm{PhCH}_{2} \mathrm{O}\right), 2.32(\mathrm{~s}, 3 \mathrm{H}, \mathrm{Mes}-p-M e)$, $2.04 \mathrm{ppm}\left(\mathrm{s}, 6 \mathrm{H}\right.$, Mes-o-Me). ${ }^{13} \mathrm{C}\left\{{ }^{1} \mathrm{H}\right\} \mathrm{NMR}\left(\mathrm{CDCl}_{3}\right): \delta=160.5$ (G0-m-Ar), 141.4 (Imz-C $\left.{ }^{2}\right), 138.2$ (ipso-Ar), 136.4 (ipso-Ph), 135.4 (p-Mes), 134.1 (o-Mes), 130.6 (ipso-Mes), 129.9 (m-Mes), 128.5 and $127.6(o-$ and $m-\mathrm{Ph}), 128.0(p-\mathrm{Ph}), 122.8\left(\mathrm{Imz}^{-} \mathrm{C}^{5}\right), 122.2\left(\mathrm{Imz}^{2} \mathrm{C}^{4}\right), 108.1$ (G0-o-Ar), 103.1 (G0-p-Ar), $70.3\left(\mathrm{PhCH}_{2} \mathrm{O}\right), 53.6\left(\mathrm{CH}_{2}\right), 21.1$ (Mes- $p$-Me), 17.7 ppm (Mes-o-Me). IR (KBr pellet): $v$ $=1610$ and $1449\left(\mathrm{~s}, \mathrm{C}-\mathrm{C}_{\text {arom }}\right), 1495(\mathrm{~m}, \mathrm{CNC}), 1263\left(\mathrm{~m}, \mathrm{C}-\mathrm{O}-\mathrm{C}_{\mathrm{as}}\right), 1155$ and $1058 \mathrm{~cm}^{-1}\left(\mathrm{~s}, \mathrm{C}-\mathrm{O}-\mathrm{C}_{\mathrm{s}}\right)$. Elemental analysis (\%) calcd for $\mathrm{C}_{33} \mathrm{H}_{33} \mathrm{BrN}_{2} \mathrm{O}_{2}$ (569.53): C 69.59, H 5.84, N 4.92; found: C 69.50, $\mathrm{H}$ 5.84, N 5.03. MS (ESI ${ }^{+}$TOF in $\left.\mathrm{CH}_{2} \mathrm{Cl}_{2} / \mathrm{MeOH} / \mathrm{NH}_{4} \mathrm{HCOO} 5 \mathrm{mM}\right): m / z=489.27[\mathrm{M}-\mathrm{Br}]^{+}$.

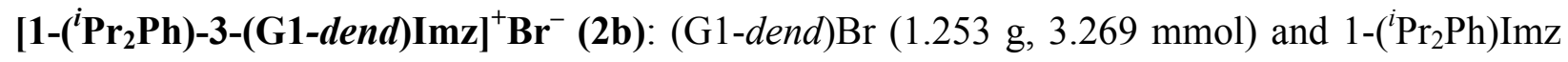
(776 mg, $3.40 \mathrm{mmol})$. Yield: $1.676 \mathrm{~g}, 84 \% .{ }^{1} \mathrm{H} \mathrm{NMR}\left(\mathrm{CDCl}_{3}\right): \delta=10.63$ (broad s, $\left.1 \mathrm{H}, \mathrm{Imz}-\mathrm{H}^{2}\right)$, $7.61\left(\mathrm{t}, J_{\mathrm{H}, \mathrm{H}}=1.6,1 \mathrm{H}, \mathrm{Imz}-\mathrm{H}^{5}\right), 7.51\left(\mathrm{t},{ }^{3} J_{\mathrm{H}, \mathrm{H}}=7.7,1 \mathrm{H}, p-{ }^{i} \mathrm{Pr}_{2} \mathrm{Ph}\right), 7.26-7.41\left(\mathrm{~m}, 12 \mathrm{H}, m-{ }^{i} \operatorname{Pr}_{2} \mathrm{Ph}\right.$ and $\mathrm{Ph}), 7.06\left(\mathrm{t}, J_{\mathrm{H}, \mathrm{H}}=1.8,1 \mathrm{H}, \mathrm{Imz}-\mathrm{H}^{4}\right), 6.84\left(\mathrm{~d},{ }^{4} J_{\mathrm{H}, \mathrm{H}}=2.2,2 \mathrm{H}, o-\mathrm{Ar}\right), 6.60\left(\mathrm{t},{ }^{4} J_{\mathrm{H}, \mathrm{H}}=2.2,1 \mathrm{H}, p-\mathrm{Ar}\right)$, $5.95\left(\mathrm{~s}, 2 \mathrm{H}, \mathrm{CH}_{2}\right), 5.02\left(\mathrm{~s}, 4 \mathrm{H}, \mathrm{PhCH}_{2} \mathrm{O}\right), 2.25\left(\mathrm{sep},{ }^{3} J_{\mathrm{H}, \mathrm{H}}=7.0,2 \mathrm{H}, \mathrm{CHMe}\right), 1.21\left(\mathrm{~d},{ }^{3} J_{\mathrm{H}, \mathrm{H}}=7.0\right.$, $6 \mathrm{H}, \mathrm{CHMe} 2), 1.10\left(\mathrm{~d},{ }^{3} J_{\mathrm{H}, \mathrm{H}}=7.0,6 \mathrm{H}, \mathrm{CHMe} 2\right) \cdot{ }^{13} \mathrm{C}\left\{{ }^{1} \mathrm{H}\right\} \mathrm{NMR}\left(\mathrm{CDCl}_{3}\right): \delta=160.5(\mathrm{G} 0-m-\mathrm{Ar})$, $145.2\left(p-{ }^{i} \mathrm{Pr}_{2} \mathrm{Ph}\right), 138.0$ (Imz-C $\left.{ }^{2}\right), 136.4$ (ipso-Ph), 135.8 (ipso-Ar), 131.8 (o- $\left.{ }^{i} \mathrm{Pr}_{2} \mathrm{Ph}\right), 130.1$ (ipso$\left.{ }^{i} \mathrm{Pr}_{2} \mathrm{Ph}\right), 128.0(p-\mathrm{Ph}), 127.6$ and 128.5 (o- and $\left.m-\mathrm{Ph}\right), 124,6\left(m-{ }^{i} \mathrm{Pr}_{2} \mathrm{Ph}\right), 123.8\left(\mathrm{Imz}-\mathrm{C}^{5}\right), 122.9$ $\left(\mathrm{Imz}-\mathrm{C}^{4}\right), 107.8$ (G0-o-Ar), 102.9 (G0-p-Ar), $70.2\left(\mathrm{Ph}-\mathrm{CH}_{2} \mathrm{O}\right), 53.4\left(\mathrm{CH}_{2}\right), 28.6\left(\mathrm{CHMe}_{2}\right), 24.4$ $\left(\mathrm{CHMe}_{2}\right), 24.0\left(\mathrm{CHMe}_{2}\right)$. IR (KBr pellet): $v=1599(\mathrm{~m})$ and 1452 (s, C-C $\left.\mathrm{Crom}\right), 1497$ (s, CNC), 1298 $\left(\mathrm{m}, \mathrm{C}-\mathrm{O}-\mathrm{C}_{\mathrm{as}}\right), 1153$ and $1058 \mathrm{~cm}^{-1}\left(\mathrm{~s}, \mathrm{C}-\mathrm{O}-\mathrm{C}_{\mathrm{s}}\right.$ ). Elemental analysis (\%) calcd for $\mathrm{C}_{36} \mathrm{H}_{39} \mathrm{BrN}_{2} \mathrm{O}_{2}$ (611.61): $\mathrm{C}$ 70.70, $\mathrm{H}$ 6.43, $\mathrm{N}$ 4.58; found: $\mathrm{C}$ 70.51, $\mathrm{H}$ 6.55, $\mathrm{N}$ 4.67. MS (ESI ${ }^{+}-\mathrm{TOF}$ in $\left.\mathrm{CH}_{2} \mathrm{Cl}_{2} / \mathrm{MeOH} / \mathrm{NH}_{4} \mathrm{HCOO} 5 \mathrm{mM}\right): m / z=531.31[\mathrm{M}-\mathrm{Br}]^{+}$.

[1-Mes-3-(G2-dend)Imz] ${ }^{+} \mathbf{B r}^{-}$(3a): (G2-dend) $\mathrm{Br}(1.219 \mathrm{~g}, 1.509 \mathrm{mmol})$ and 1-(Mes)Imz (300 mg, $1.61 \mathrm{mmol})$. Yield: $1.30 \mathrm{~g}, 87 \% .{ }^{1} \mathrm{H}$ NMR $\left(\mathrm{CDCl}_{3}\right): \delta=10.65$ (s, 1H, Imz-H²), 7.40-7.26 (m, $21 \mathrm{H}, \mathrm{Ph}$ and $\left.\mathrm{Imz}-\mathrm{H}^{5}\right), 6.93(\mathrm{~s}, 2 \mathrm{H}, m-\mathrm{Mes}), 6.90\left(\mathrm{~s}, 1 \mathrm{H}, \mathrm{Imz}_{-} \mathrm{H}^{4}\right), 6.80\left(\mathrm{~d},{ }^{4} J_{\mathrm{H}, \mathrm{H}}=2.0 \mathrm{~Hz}, 2 \mathrm{H}, \mathrm{G} 0-o-\right.$ Ar), $6.66\left(\mathrm{~d},{ }^{4} J_{\mathrm{H}, \mathrm{H}}=2.0 \mathrm{~Hz}, 4 \mathrm{H}, \mathrm{G} 1-o-\mathrm{Ar}\right), 6.57\left(\mathrm{t},{ }^{4} J_{\mathrm{H}, \mathrm{H}}=2.0 \mathrm{~Hz}, 1 \mathrm{H}, \mathrm{G} 0-p-\mathrm{Ar}\right), 6.53\left(\mathrm{t},{ }^{4} J_{\mathrm{H}, \mathrm{H}}=2.0\right.$ $\mathrm{Hz}, 2 \mathrm{H}, \mathrm{G} 1-p-\mathrm{Ar}), 5.84$ (s, 2H, $\left.\mathrm{CH}_{2}\right), 5.00$ (s, 8H, $\left.\mathrm{PhCH}_{2} \mathrm{O}\right), 4.98$ (s, 4H G1-CH$\left.{ }_{2} \mathrm{O}\right), 2.30(\mathrm{~s}, 3 \mathrm{H}$, Mes-p-Me), 2.01 ppm (s, 6H, Mes-o-Me). ${ }^{13} \mathrm{C}\left\{{ }^{1} \mathrm{H}\right\}$ NMR $\left(\mathrm{CDCl}_{3}\right): \delta=160.3(\mathrm{G} 0-m-\mathrm{Ar}), 160.0$ (G1-m-Ar), 141.4 (Imz-C ${ }^{2}$ ), 138.9 (G1-ipso-Ar), 137.9 (G0-ipso-Ar), 136.7 (ipso-Ph), 135.4 (pMes), 134.0 (o-Mes), 130.5 (ipso-Mes), 129.8 (m-Mes), 128.5 and 127.5 (o- and $m$-Ph), 127.9 ( $p$ $\mathrm{Ph}), 122.8\left(\mathrm{Imz}^{-C^{5}}\right), 122.5$ (Imz-C $\left.{ }^{4}\right), 108.0$ (G0-o-Ar), 106.4 (G1-o-Ar), 103.1 (G0-p-Ar), 101.5 (G1-p-Ar), $70.0\left(\mathrm{PhCH}_{2} \mathrm{O}\right.$ and $\left.\mathrm{G} 1-\mathrm{CH}_{2} \mathrm{O}\right), 53.4\left(\mathrm{CH}_{2}\right), 21.0$ (Mes-p-Me), 17.6 ppm (Mes-o-Me). IR (KBr pellet): $v=1594$ and 1452 (vs, C-C arom), 1497 (m, CNC), 1294 (m, C-O-C $\mathrm{as}_{\text {s) }}, 1160$ and 1051 $\mathrm{cm}^{-1}$ (vs, C-O-C ). Elemental analysis (\%) calcd for $\mathrm{C}_{61} \mathrm{H}_{57} \mathrm{BrN}_{2} \mathrm{O}_{6}(994.02)$ : C 73.71, H 5.78, N 2.82; found: $\mathrm{C}$ 73.34, $\mathrm{H}$ 5.82, $\mathrm{N}$ 2.91. $\mathrm{MS}\left(\mathrm{ESI}^{+}-\mathrm{TOF}\right.$ in $\left.\mathrm{CH}_{2} \mathrm{Cl}_{2} / \mathrm{MeOH} / \mathrm{NH}_{4} \mathrm{HCOO} 5 \mathrm{mM}\right): \mathrm{m} / z=$ $913.43[\mathrm{M}-\mathrm{Br}]^{+}$. 


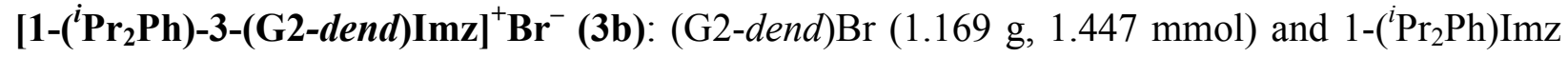
(365 mg, $1.60 \mathrm{mmol})$. Yield: $1.262 \mathrm{~g}, 84 \% .{ }^{1} \mathrm{H}$ NMR $\left(\mathrm{CDCl}_{3}\right): \delta=10.71$ (broad s, $\left.1 \mathrm{H}, \mathrm{Imz}-\mathrm{H}^{2}\right)$, $7.52\left(\mathrm{t}, J_{H, H}=1.6,1 \mathrm{H}, \mathrm{Imz}-\mathrm{H}^{5}\right), 7.50\left(\mathrm{t},{ }^{3} J_{\mathrm{H}, \mathrm{H}}=7.9,1 \mathrm{H}, p-{ }^{i} \mathrm{Pr}_{2} \mathrm{Ph}\right), 7.25-7.40\left(\mathrm{~m}, 22 \mathrm{H}, m-{ }^{i} \operatorname{Pr}_{2} \mathrm{Ph}\right.$ and $\mathrm{Ph}), 7.00\left(\mathrm{t}, J_{H, H}=1.6,1 \mathrm{H}, \mathrm{Imz}-\mathrm{H}^{4}\right), 6.82\left(\mathrm{~d},{ }^{4} J_{\mathrm{H}, \mathrm{H}}=2.2,2 \mathrm{H}, \mathrm{G} 0-o-\mathrm{Ar}\right), 6.65\left(\mathrm{~d},{ }^{4} J_{\mathrm{H}, \mathrm{H}}=2.2,4 \mathrm{H}\right.$, G1-o-Ar), 6.55 (m, 3H, G0-p-Ar and G1-p-Ar), 5.94 (s, 2H, $\left.\mathrm{CH}_{2}\right), 5.01$ (s, 8H, $\left.\mathrm{PhCH}_{2} \mathrm{O}\right), 4.96$ (s, $\left.4 \mathrm{H}, \mathrm{G} 1-\mathrm{CH}_{2} \mathrm{O}\right), 2.25\left(\mathrm{sep},{ }^{3} J_{\mathrm{H}, \mathrm{H}}=6.8,2 \mathrm{H}, \mathrm{CHMe} 2\right), 1.21\left(\mathrm{~d},{ }^{3} J_{\mathrm{H}, \mathrm{H}}=6.8,6 \mathrm{H}, \mathrm{CHMe} e_{2}\right), 1.10\left(\mathrm{~d},{ }^{3} J_{\mathrm{H}, \mathrm{H}}\right.$ $\left.=6.8,6 \mathrm{H}, \mathrm{CHMe} e_{2}\right) \cdot{ }^{13} \mathrm{C}\left\{{ }^{1} \mathrm{H}\right\} \mathrm{NMR}\left(\mathrm{CDCl}_{3}\right): \delta=160.4(\mathrm{G} 0-m-\mathrm{Ar}), 160.1$ (G1-m-Ar), $145.2(p-$ $\left.{ }^{i} \mathrm{Pr}_{2} \mathrm{Ph}\right), 138.8$ (G1-ipso-Ar), 138.4 (Imz-C $\left.{ }^{2}\right), 136.7$ (ipso-Ph), 135.7 (G0-ipso-Ar), 131.9 (o- $\left.{ }^{i} \mathrm{Pr}_{2} \mathrm{Ph}\right)$, $130.0\left(\right.$ ipso- $\left.{ }^{i} \mathrm{Pr}_{2} \mathrm{Ph}\right), 128.0(p-\mathrm{Ph}), 127.5$ and $128.5(o-$ and $m-\mathrm{Ph}), 124.6\left(m-{ }^{i} \mathrm{Pr} 2 \mathrm{Ph}\right), 123.8\left(\mathrm{Imz}^{-C^{5}}\right)$, 122.6 (Imz-C ${ }^{4}$ ), 107.8 (G0-o-Ar), 106.4 (G1-p-Ar), 102.9 (G0-p-Ar), 101.5 (G1-p-Ar), 70.0 (Ph$\mathrm{CH}_{2} \mathrm{O}$ and $\mathrm{Ar}-\mathrm{CH}_{2} \mathrm{O}$ overlapping), $53.5\left(\mathrm{CH}_{2}\right), 28.7\left(\mathrm{CHMe}_{2}\right), 24.4(\mathrm{CHMe}), 24.0(\mathrm{CHMe})$. IR (KBr pellet): $v=1599(\mathrm{~m})$ and 1452 (s, C-C arom), 1497 (s, CNC), 1297 (m, C-O-Cas), 1154 and $1052 \mathrm{~cm}^{-1}$ (s, C-O-C ). Elemental analysis (\%) calcd for $\mathrm{C}_{64} \mathrm{H}_{63} \mathrm{BrN}_{2} \mathrm{O}_{6}$ (1036.10): C 74.19, H 6.13, $\mathrm{N}$ 2.70; found: $\mathrm{C} 74.00, \mathrm{H}$ 6.26, N 2.72. MS (ESI ${ }^{+}-\mathrm{TOF}$ in $\left.\mathrm{CH}_{2} \mathrm{Cl}_{2} / \mathrm{MeOH} / \mathrm{NH}_{4} \mathrm{HCOO} 5 \mathrm{mM}\right): m / z=$ $955.49[\mathrm{M}-\mathrm{Br}]^{+}$.

[1-Mes-3-(G3-dend)Imz] ${ }^{+} \mathbf{B r}^{-}$(4a): (G3-dend) $\mathrm{Br}(1.20 \mathrm{~g}, 0.724 \mathrm{mmol})$ and 1-(Mes)Imz (138 mg, $0.741 \mathrm{mmol})$. Yield: $1.14 \mathrm{~g}, 85 \%$. ${ }^{1} \mathrm{H}$ NMR $\left(\mathrm{CDCl}_{3}\right): \delta=10.70$ (s, 1H, Imz-H $\left.{ }^{2}\right), 7.40-7.26(\mathrm{~m}$, $41 \mathrm{H}, \mathrm{Ph}$ and $\left.\mathrm{Imz}-\mathrm{H}^{5}\right), 6.93(\mathrm{~s}, 2 \mathrm{H}, m-\mathrm{Mes}), 6.90\left(\mathrm{~s}, 1 \mathrm{H}, \mathrm{Imz}_{-} \mathrm{H}^{4}\right), 6.81\left(\mathrm{~d},{ }^{4} J_{\mathrm{H}, \mathrm{H}}=2.0 \mathrm{~Hz}, 2 \mathrm{H}, \mathrm{G} 0-o-\right.$ $\mathrm{Ar}), 6.67\left(\mathrm{~d},{ }^{4} J_{\mathrm{H}, \mathrm{H}}=2.2 \mathrm{~Hz}, 8 \mathrm{H}, \mathrm{G} 2-\mathrm{o}-\mathrm{Ar}\right), 6.66\left(\mathrm{~d},{ }^{4} J_{\mathrm{H}, \mathrm{H}}=2.2 \mathrm{~Hz}, 4 \mathrm{H}, \mathrm{G} 1-o-\mathrm{Ar}\right), 6.61\left(\mathrm{t},{ }^{4} J_{\mathrm{H}, \mathrm{H}}=2.2\right.$ $\mathrm{Hz}, 1 \mathrm{H}, \mathrm{G} 0-p-\mathrm{Ar}), 6.55\left(\mathrm{t},{ }^{4} J_{\mathrm{H}, \mathrm{H}}=2.2 \mathrm{~Hz}, 4 \mathrm{H}, \mathrm{G} 2-p-\mathrm{Ar}\right), 6.52\left(\mathrm{t},{ }^{4} J_{\mathrm{H}, \mathrm{H}}=2.2 \mathrm{~Hz}, 2 \mathrm{H}, \mathrm{G} 1-p-\mathrm{Ar}\right), 5.81$ (s, 2H, $\mathrm{CH}_{2}$ ), 5.00 (s, 20H G1- $\mathrm{CH}_{2} \mathrm{O}$ and $\left.\mathrm{PhCH} \mathrm{H}_{2} \mathrm{O}\right), 4.96\left(\mathrm{~s}, 8 \mathrm{H}, \mathrm{G} 2-\mathrm{CH}_{2} \mathrm{O}\right), 2.28(\mathrm{~s}, 3 \mathrm{H}$, Mes- $p$ $\mathrm{Me}$ ), $2.00 \mathrm{ppm}\left(\mathrm{s}, 6 \mathrm{H}\right.$, Mes-o-Me). ${ }^{13} \mathrm{C}\left\{{ }^{1} \mathrm{H}\right\} \mathrm{NMR}\left(\mathrm{CDCl}_{3}\right): \delta=160.4$ (G0-m-Ar), 160.1 (G2-m-Ar), 160.0 (G1-m-Ar), 141.3 (Imz-C ${ }^{2}$ ), 139.2 (G2-ipso-Ar), 138.9 (G1-ipso-Ar), 138.2 (G0-ipso-Ar), 136.7 (ipso-Ph), 135.3 (p-Mes), 134.1 (o-Mes), 130.5 (ipso-Mes), 129.8 (m-Mes), 128.0 (p-Ph), 128.5 and 127.5 (o- and $m-\mathrm{Ph}), 122.7\left(\mathrm{Imz}^{-} \mathrm{C}^{5}\right), 122.1$ (Imz-C $\left.{ }^{4}\right), 108.0$ (G0-o-Ar), 106.4 (G1-o-Ar), 106.3 (G2-o-Ar), 103.1 (G0-p-Ar), 101.6 (G1-p-Ar), 101.5 (G2-p-Ar), 70.0 and 70.1 (PhCH $\mathrm{O}, \mathrm{G} 1$ $\mathrm{CH}_{2} \mathrm{O}$ and $\left.\mathrm{G} 2-\mathrm{CH}_{2} \mathrm{O}\right), 53.5\left(\mathrm{CH}_{2}\right), 21.0($ Mes- $p-M e), 17.6$ ppm (Mes-o-Me). IR (KBr pellet): $v=$ 1599 and 1447 (vs, C-C arom), 1497 (m, CNC), 1295 (s, C-O-C $\mathrm{Cas}_{\mathrm{as}}$ ), 1147 and $1048 \mathrm{~cm}^{-1}$ (vs, C-O-C $\mathrm{C}_{\mathrm{s}}$ ). Elemental analysis (\%) calcd for $\mathrm{C}_{117} \mathrm{H}_{105} \mathrm{BrN}_{2} \mathrm{O}_{14}$ (1842.99): $\mathrm{C} 76.25$, H 5.74, N 1.52; found: C 75.95, $\mathrm{H}$ 5.80, N 1.58. MS (ESI ${ }^{+}$-TOF in $\left.\mathrm{CH}_{2} \mathrm{Cl}_{2} / \mathrm{MeOH} / \mathrm{NH}_{4} \mathrm{HCOO} 5 \mathrm{mM}\right): \mathrm{m} / z=1761.76[\mathrm{M}-$ $\mathrm{Br}]^{+}$.

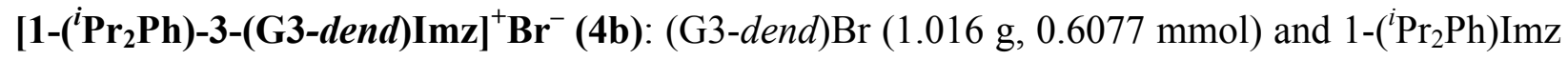
(142 mg, $0.622 \mathrm{mmol})$. Yield: $1.110 \mathrm{~g}, 97 \% .{ }^{1} \mathrm{H}$ NMR $\left(\mathrm{CDCl}_{3}\right): \delta=10.72\left(\right.$ broad s, $\left.1 \mathrm{H}, \mathrm{Imz}-\mathrm{H}^{2}\right)$, 7.44-7.46 (m, 2H, $p-{ }^{i} \operatorname{Pr}_{2} \mathrm{Ph}$ and $\left.\mathrm{Imz}_{-} \mathrm{H}^{5}\right)$, 7.24-7.40 (m, 42H, $m-{ }^{i}{ }^{2}{ }_{2} \mathrm{Ph}$ and $\mathrm{Ph}$ ), 6.98 (virtual $\mathrm{t}, J_{\mathrm{H}, \mathrm{H}}=$ 1.6, 1H, Imz-H $\left.{ }^{4}\right), 6.82\left(\mathrm{~d},{ }^{4} J_{\mathrm{H}, \mathrm{H}}=2.0,2 \mathrm{H}, \mathrm{G} 0-\mathrm{o}-\mathrm{Ar}\right), 6.65(\mathrm{~m}, 12 \mathrm{H}, \mathrm{G} 1-\mathrm{o}-\mathrm{Ar}$ and G2-o-Ar), 6.59 (t, 
$\left.{ }^{4} J_{\mathrm{H}, \mathrm{H}}=2.0,1 \mathrm{H}, \mathrm{G} 0-o-\mathrm{Ar}\right), 6.53(\mathrm{~m}, 6 \mathrm{H}, \mathrm{G} 1-p-\mathrm{Ar}$ and $\mathrm{G} 2-p-\mathrm{Ar}), 4.99\left(\mathrm{~s}, 16 \mathrm{H}, \mathrm{PhCH}_{2} \mathrm{O}\right), 5.91(\mathrm{~s}$, $\left.2 \mathrm{H}, \mathrm{CH}_{2}\right), 4.94\left(\mathrm{~s}, 12 . \mathrm{H}, \mathrm{G} 1-\mathrm{CH}_{2} \mathrm{O}\right.$ and $\left.\mathrm{G} 2-\mathrm{CH}_{2} \mathrm{O}\right), 2.23\left(\mathrm{sep},{ }^{3} \mathrm{~J}_{\mathrm{H}, \mathrm{H}}=6.9,2 \mathrm{H}, \mathrm{CHMe}\right.$ ), $1.20(\mathrm{~d}$, $\left.{ }^{3} J_{\mathrm{H}, \mathrm{H}}=6.9,6 \mathrm{H}, \mathrm{CHMe} 2\right), 1.08\left(\mathrm{~d},{ }^{3} J_{\mathrm{H}, \mathrm{H}}=6.9,6 \mathrm{H}, \mathrm{CHMe} 2\right) .{ }^{13} \mathrm{C}\left\{{ }^{1} \mathrm{H}\right\} \mathrm{NMR}\left(\mathrm{CDCl}_{3}\right): \delta=160.5(\mathrm{G} 0-$ m-Ar), 160.1 (G2-m-Ar), 160.0 (G1-m-Ar), 145.2 ( $p$ - $\left.{ }^{i} \mathrm{Pr}_{2} \mathrm{Ph}\right), 139.2$ (G2-ipso-Ar), 138.8 (G1-ipsoAr), 138.5 (Imz-C $\left.{ }^{2}\right), 136.7$ (ipso-Ph), 131.9 (o- $\left.{ }^{i} \mathrm{Pr}_{2} \mathrm{Ph}\right), 131.7$ (G0-ipso-Ar), 130.0 (ipso- $\left.{ }^{i} \mathrm{Pr}_{2} \mathrm{Ph}\right)$, $128.0(p-\mathrm{Ph}), 127.5$ and $128.5(o-$ and $m-\mathrm{Ph}), 124.7\left(m-{ }^{i} \mathrm{Pr}_{2} \mathrm{Ph}\right), 123.8\left(\mathrm{Imz}_{-} \mathrm{C}^{5}\right), 122.4\left(\mathrm{Imz}-\mathrm{C}^{4}\right)$, 107.9 (G0-o-Ar), 106.5 (G1-p-Ar), 106.4 (G2-p-Ar), 102.8 (G0-p-Ar), 101.6 (G1-p-Ar), 101.5 (G2p-Ar), $70.0\left(\mathrm{Ph}-\mathrm{CH}_{2} \mathrm{O}, \mathrm{G} 1-\mathrm{Ar}-\mathrm{CH}_{2} \mathrm{O}\right.$ and $\mathrm{G} 0-\mathrm{Ar}-\mathrm{CH}_{2} \mathrm{O}$ overlapping), $53.6\left(\mathrm{CH}_{2}\right), 28.7\left(\mathrm{CHMe}_{2}\right)$, $24.4\left(\mathrm{CHMe}_{2}\right), 24.0(\mathrm{CHMe})$. IR (KBr pellet): $v=1607$ (m) and 1448 (s, C-Carom), 1497 (s, CNC), $1296\left(\mathrm{~m}, \mathrm{C}-\mathrm{O}-\mathrm{C}_{\mathrm{as}}\right), 1154$ and $1055 \mathrm{~cm}^{-1}$ (s, C-O-C $\left.\mathrm{C}_{\mathrm{s}}\right)$. Elemental analysis (\%) calcd for $\mathrm{C}_{120} \mathrm{H}_{111} \mathrm{BrN}_{2} \mathrm{O}_{14}$ (1885.07): C 76.46, H 5.94, N 1.49; found: C 75.89, H 5.91, N 1.48. MS (ESI TOF in $\left.\mathrm{CH}_{2} \mathrm{Cl}_{2} / \mathrm{MeOH} / \mathrm{NH}_{4} \mathrm{HCOO} 5 \mathrm{mM}\right): m / z=1803.80[\mathrm{M}-\mathrm{Br}]^{+}$. 


\section{Preparation and characterization data for NHC-Ag complexes $5 a-8 a$ and $5 b-8 b$}

$\mathrm{Ag}_{2} \mathrm{O}$ was added to an Schlenk tube containing a $\mathrm{CH}_{2} \mathrm{Cl}_{2}(20-30 \mathrm{~mL})$ solution of an imidazolium salt $1 \mathbf{a}-\mathbf{4 a}$ or $\mathbf{1 b}-\mathbf{4 b}$. In order to allow the reaction to complete the mixture was stirred in the dark at room temperature 16 h, 3 days, 4 days, and 7 days, for $\mathbf{5 a}, \mathbf{6 a}, \mathbf{7 a}$, and $\mathbf{8 a}$, or 1, 1, 4, and 8 days, for $\mathbf{5 b}, \mathbf{6 b}, \mathbf{7 b}$, and $\mathbf{8 b}$, respectively. In some cases, an excess of $\mathrm{Ag}_{2} \mathrm{O}$ was added at the specified time. The silver compounds were obtained as off-white solids (white in the case of 5a) after filtration of the $\mathrm{Ag}_{2} \mathrm{O}$ excess, evaporation of the solvent under vacuum to dryness, and washings with hexane (3 $\times 20 \mathrm{~mL})$, and additional washings with diethyl ether $(3 \times 20 \mathrm{~mL})$ in the case of $\mathbf{8 b}$.

[AgBr\{1-Mes-3-(G0-dend)Imz-2-ylidene\}] (5a): $\mathrm{Ag}_{2} \mathrm{O}$ (194 mg, $\left.0.837 \mathrm{mmol}\right)$ and 1a (600 mg, $1.68 \mathrm{mmol})$. Yield: $758 \mathrm{mg}, 97 \% .{ }^{1} \mathrm{H}$ NMR $\left(\mathrm{CDCl}_{3}\right): \delta=7.39-7.34(\mathrm{~m}, 3 \mathrm{H}, \mathrm{Ph}), 7.27-7.22(\mathrm{~m}, 2 \mathrm{H}$, $\mathrm{Ph}), 7.08\left(\mathrm{~d},{ }^{3} J_{\mathrm{H}, \mathrm{H}}=1.6,1 \mathrm{H}, \mathrm{Imz}-\mathrm{H}^{5}\right), 6.94(\mathrm{~s}, 2 \mathrm{H}, m-\mathrm{Mes}), 6.92\left(\mathrm{~d},{ }^{3} J_{\mathrm{H}, \mathrm{H}}=1.6,1 \mathrm{H}, \mathrm{Imz}-\mathrm{H}^{4}\right), 5.39$ $\left(\mathrm{s}, 2 \mathrm{H}, \mathrm{CH}_{2}\right), 2.32(\mathrm{~s}, 3 \mathrm{H}, \mathrm{Mes}-p-M e), 1.97 \mathrm{ppm}(\mathrm{s}, 6 \mathrm{H}, \mathrm{Mes}-o-M e) .{ }^{13} \mathrm{C}\left\{{ }^{1} \mathrm{H}\right\} \mathrm{NMR}\left(\mathrm{CDCl}_{3}\right): \delta=$ 139.6 (p-Mes), 135.5 (ipso-Ph), 135.2 (ipso-Mes), 134.6 (o-Mes), 129.5 (o-Ph), 129.2 (m-Mes), $128.7(p-\mathrm{Ph}), 127.5(m-\mathrm{Ph}), 123.2\left(\mathrm{Imz}^{-} \mathrm{C}^{5}\right), 121.0\left(\mathrm{Imz}^{-} \mathrm{C}^{4}\right), 55.8\left(\mathrm{CH}_{2}\right), 21.0(\mathrm{Mes}-p-\mathrm{Me}), 17.7$ ppm (Mes-o-Me), Imz-C $\mathrm{C}^{2}$ not observed. IR (KBr pellet): $v=1607(\mathrm{~m})$ and 1455 (s, C-C $\left.\mathrm{C}_{\mathrm{arom}}\right), 1495$ $\mathrm{cm}^{-1}$ (s, CNC). Elemental analysis (\%) calcd for $\mathrm{C}_{19} \mathrm{H}_{20} \mathrm{AgBrN}_{2}$ (464.15): C 49.17, H 4.34, N 6.04; found: $\mathrm{C} 49.41, \mathrm{H}$ 4.35, $\mathrm{N}$ 6.22. $\mathrm{MS}$ (ESI ${ }^{+}$TOF in $\mathrm{CH}_{2} \mathrm{Cl}_{2} / \mathrm{MeOH} / \mathrm{NH}_{4} \mathrm{HCOO} 5 \mathrm{~mm}$ ): $\mathrm{m} / z=659.23$ $\left[\mathrm{M}_{2}-\mathrm{AgBr}_{2}\right]^{+}, 277.17[\mathbf{1 a}-\mathrm{Br}]^{+}$

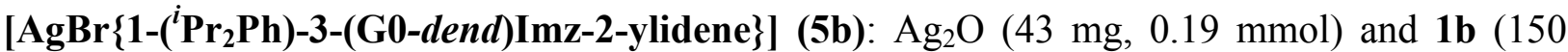
$\mathrm{mg}, 0.376 \mathrm{mmol})$. Yield after recrystallization in $\mathrm{CH}_{2} \mathrm{Cl}_{2} /$ hexane: $140 \mathrm{mg}, 75 \% .{ }^{1} \mathrm{H} \mathrm{NMR}\left(\mathrm{CDCl}_{3}\right)$ : $\delta=7.20-7.50\left(\mathrm{~m}, 8 \mathrm{H},{ }^{i} \mathrm{Pr}_{2} \mathrm{Ph}\right.$ and $\left.\mathrm{Ph}\right), 7.08\left(\mathrm{~d},{ }^{3} J_{\mathrm{H}, \mathrm{H}}=1.3,1 \mathrm{H}, \mathrm{Imz}-\mathrm{H}^{5}\right), 6.97\left(\mathrm{~d},{ }^{3} J_{\mathrm{H}, \mathrm{H}}=1.3,1 \mathrm{H}\right.$, Imz-H $\left.{ }^{4}\right), 5.41\left(\mathrm{~s}, 2 \mathrm{H}, \mathrm{CH}_{2}\right), 2.37\left(\mathrm{sep},{ }^{3} J_{\mathrm{H}, \mathrm{H}}=6.9,2 \mathrm{H}, \mathrm{CHMe} 2\right), 1.23\left(\mathrm{~d},{ }^{3} J_{\mathrm{H}, \mathrm{H}}=6.9,6 \mathrm{H}, \mathrm{CHMe}\right)_{2}$, $\left.1.10\left(\mathrm{~d},{ }^{3} J_{\mathrm{H}, \mathrm{H}}=6.9,6 \mathrm{H}, \mathrm{CHMe}\right)_{2}\right) .{ }^{13} \mathrm{C}\left\{{ }^{1} \mathrm{H}\right\} \mathrm{NMR}\left(\mathrm{CDCl}_{3}\right): \delta=145.6\left(p-{ }^{i} \mathrm{Pr}_{2} \mathrm{Ph}\right), 135.3$ (ipso-Ph), 134.5 (ipso- ${ }^{i} \mathrm{Pr}_{2} \mathrm{Ph}$ ), $130.6\left(o-{ }^{i} \mathrm{Pr}_{2} \mathrm{Ph}\right), 128.8$ ( $p$ - $\left.\mathrm{Ph}\right), 127.6$ and 129.2 (o- and $m-\mathrm{Ph}$ overlapping), 124.2 (Imz-C ${ }^{5}$ and $m-{ }^{i} \mathrm{Pr}_{2} \mathrm{Ph}$ overlapping), $120.7\left(\mathrm{~d},{ }^{3} J_{\mathrm{C}, \mathrm{Ag}} \approx 4.8, \mathrm{Imz}-\mathrm{C}^{4}\right), 52.9\left(\mathrm{CH}_{2}\right), 28.4$ $\left(\mathrm{CHMe}_{2}\right), 24.6\left(\mathrm{CHMe}_{2}\right), 24.3\left(\mathrm{CHMe}_{2}\right)$, Imz-C ${ }^{2}$ not observed. IR (KBr pellet): $v=1593$ and 1456 (s, C-C $\mathrm{C}_{\text {arom}}$ ), $1496 \mathrm{~cm}^{-1}$ (m, CNC). Elemental analysis (\%) calcd for $\mathrm{C}_{22} \mathrm{H}_{26} \mathrm{AgBrN}_{2}$ (506.23): C 52.20, $\mathrm{H}$ 5.18, $\mathrm{N}$ 5.53; found: $\mathrm{C} 52.18, \mathrm{H}$ 5.06, $\mathrm{N}$ 5.50. MS $\left(\mathrm{ESI}^{+}-\mathrm{TOF}\right.$ in $\left.\mathrm{CH}_{2} \mathrm{Cl}_{2} / \mathrm{MeOH} / \mathrm{NH}_{4} \mathrm{HCOO} 5 \mathrm{mM}\right): m / z=743.33\left[\mathrm{M}_{2}-\mathrm{AgBr}_{2}\right]^{+}, 425.11[\mathrm{M}-\mathrm{Br}]^{+}, 319.23[\mathbf{1 b}-$ $\mathrm{Br}]^{+}$.

[AgBr\{1-Mes-3-(G1-dend)Imz-2-ylidene\}] (6a): $\mathrm{Ag}_{2} \mathrm{O}(70 \mathrm{mg}, 0.30 \mathrm{mmol})$ and 2a (335 mg, $0.588 \mathrm{mmol})$. Yield: $374 \mathrm{mg}, 94 \% .{ }^{1} \mathrm{H}$ NMR $\left(\mathrm{CDCl}_{3}\right): \delta=7.41-7.29(\mathrm{~m}, 10 \mathrm{H}, \mathrm{Ph}), 7.08\left(\mathrm{~d},{ }^{3} J_{\mathrm{H}, \mathrm{H}}=\right.$ 1.6, $\left.1 \mathrm{H}, \mathrm{Imz}-\mathrm{H}^{5}\right), 6.98(\mathrm{~s}, 2 \mathrm{H}, m-\mathrm{Mes}), 6.90\left(\mathrm{~d},{ }^{3} J_{\mathrm{H}, \mathrm{H}}=1.6,1 \mathrm{H}, \mathrm{Imz}-\mathrm{H}^{4}\right), 6.58\left(\mathrm{t},{ }^{4} J_{\mathrm{H}, \mathrm{H}}=2.2,1 \mathrm{H}, p-\right.$ 
Ar), $6.48\left(\mathrm{~d},{ }^{4} J_{\mathrm{H}, \mathrm{H}}=2.2,2 \mathrm{H}, o-\mathrm{Ar}\right), 5.29\left(\mathrm{~s}, 2 \mathrm{H}, \mathrm{CH}_{2}\right), 5.02\left(\mathrm{~s}, 4 \mathrm{H}, \mathrm{PhCH} \mathrm{H}_{2} \mathrm{O}\right), 2.31(\mathrm{~s}, 3 \mathrm{H}, \mathrm{Mes}-p$ Me), 1.96 ppm (s, 6H, Mes-o-Me). ${ }^{13} \mathrm{C}\left\{{ }^{1} \mathrm{H}\right\} \mathrm{NMR}\left(\mathrm{CDCl}_{3}\right): \delta=160.5$ (G0-m-Ar), 139.6 (p-Mes), 137.1 (ipso-Ar), 136.4 (ipso -Ph), 135.2 (ipso-Mes), 134.6 (o-Mes), 129.4 (m-Mes), 128.6 and $127.5(o-$ and $m-\mathrm{Ph}), 128.1(p-\mathrm{Ph}), 123.0\left(\mathrm{Imz}^{-} \mathrm{C}^{5}\right), 121.2\left(\mathrm{Imz}^{4} \mathrm{C}^{4}\right), 106.8$ (G0-o-Ar), 102.1 (G0-pAr), $70.2\left(\mathrm{PhCH}_{2} \mathrm{O}\right), 55.7\left(\mathrm{CH}_{2}\right), 21.0$ (Mes-p-Me), 17.7 ppm (Mes-o-Me), Imz-C not observed. IR (KBr pellet): $v=1595$ and 1449 (vs, C-C $\mathrm{C}_{\text {arom }}$ ), 1496 (s, CNC), 1295 (m, C-O-Cas), 1160 and 1057 $\mathrm{cm}^{-1}$ (vs, C-O-C ${ }_{\mathrm{s}}$ ). Elemental analysis (\%) calcd for $\mathrm{C}_{33} \mathrm{H}_{32} \mathrm{AgBrN}_{2} \mathrm{O}_{2}$ (676.39): C 58.60, H 4.77, $\mathrm{N}$ 4.14; found: $\mathrm{C} 59.05, \mathrm{H} 4.71, \mathrm{~N}$ 4.27. $\mathrm{MS}\left(\mathrm{ESI}^{+}-\mathrm{TOF}\right.$ in $\left.\mathrm{CH}_{2} \mathrm{Cl}_{2} / \mathrm{MeOH} / \mathrm{NH}_{4} \mathrm{HCOO} 5 \mathrm{mM}\right): \mathrm{m} / z=$ $1083.40\left[\mathrm{M}_{2}-\mathrm{AgBr}_{2}\right]^{+}, 489.25[\mathbf{2 a}-\mathrm{Br}]^{+}$.

[AgBr\{1-( $\left.\left.\left.{ }^{i} \mathbf{P r}_{2} \mathbf{P h}\right)-3-(G 1-d e n d) I m z-2-y l i d e n e\right\}\right] ~(6 b): ~ g_{2} \mathrm{O}$ (115 mg, $\left.0.496 \mathrm{mmol}\right)$ and $2 \mathbf{b}$ (600 $\mathrm{mg}, 0.981 \mathrm{mmol})$ and. Yield after recrystallization in $\mathrm{CH}_{2} \mathrm{Cl}_{2} /$ hexane: $580 \mathrm{mg}, 82 \%$. ${ }^{1} \mathrm{H} \mathrm{NMR}$ $\left(\mathrm{CDCl}_{3}\right): \delta=7.26-7.41\left(\mathrm{~m}, 12 \mathrm{H},{ }^{i} \mathrm{Pr}_{2} \mathrm{Ph}\right.$ and $\left.\mathrm{Ph}\right), 7.08$ (broad s, Imz-H $\left.{ }^{5}\right), 6.98$ (broad s, Imz-H ${ }^{4}$ ), $6.58\left(\mathrm{t},{ }^{4} J_{\mathrm{H}, \mathrm{H}}=2.2,1 \mathrm{H}, p-\mathrm{Ar}\right), 6.47\left(\mathrm{~d},{ }^{4} J_{\mathrm{H}, \mathrm{H}}=2.2,2 \mathrm{H}, o-\mathrm{Ar}\right), 5.33\left(\mathrm{~s}, 2 \mathrm{H}, \mathrm{CH}_{2}\right), 5.00(\mathrm{~s}, 4 \mathrm{H}$, $\left.\mathrm{PhCH}_{2} \mathrm{O}\right), 2.38\left(\mathrm{sep},{ }^{3} J_{\mathrm{H}, \mathrm{H}}=6.6,2 \mathrm{H}, \mathrm{CHMe}\right), 1.21\left(\mathrm{~d},{ }^{3} J_{\mathrm{H}, \mathrm{H}}=6.6,6 \mathrm{H}, \mathrm{CHMe} e_{2}\right), 1.08\left(\mathrm{~d},{ }^{3} J_{\mathrm{H}, \mathrm{H}}=6.6\right.$, $6 \mathrm{H}, \mathrm{CHMe} 2) .{ }^{13} \mathrm{C}\left\{{ }^{1} \mathrm{H}\right\}$ NMR $\left(\mathrm{CDCl}_{3}\right): \delta=160.6$ (G0-m-Ar), $145.6\left(p-{ }^{i} \mathrm{Pr}{ }_{2} \mathrm{Ph}\right), 137.7$ (ipso-Ar), 136.4 (ipso-Ph), 134.6 (ipso- $\left.{ }^{i} \mathrm{Pr}_{2} \mathrm{Ph}\right), 130.6\left(o-{ }^{i}{ }^{\mathrm{Pr}}{ }_{2} \mathrm{Ph}\right), 128.1$ (p-Ph), 127.6 and 128.6 (o- and $\left.m-\mathrm{Ph}\right)$, 124.3 (Imz-C ${ }^{5}$ and $m-{ }^{i}{ }^{P} r_{2} \mathrm{Ph}$ overlapping), $120.9\left({ }^{3} J_{\mathrm{C}, \mathrm{Ag}} \approx 8.4, \mathrm{Imz} \mathrm{C}^{4}\right), 106.7$ (G0-o-Ar), 102.1 (G0p-Ar), $70.3\left(\mathrm{Ph}-\mathrm{CH}_{2} \mathrm{O}\right), 55.8\left(\mathrm{CH}_{2}\right), 28.4\left(\mathrm{CHMe}_{2}\right), 24.6\left(\mathrm{CHMe}_{2}\right), 24.3(\mathrm{CHMe}), \mathrm{Imz}-\mathrm{C}^{2}$ not

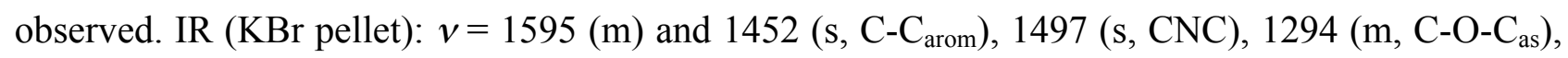
$1058 \mathrm{~cm}^{-1}$ (s, C-O-C $\mathrm{C}_{\mathrm{s}}$ ). Elemental analysis (\%) calcd for $\mathrm{C}_{36} \mathrm{H}_{38} \mathrm{AgBrN}_{2} \mathrm{O}_{2}$ (718.47): C 60.18, $\mathrm{H}$ 5.33, N 3.90; found: $\mathrm{C}$ 60.42, $\mathrm{H}$ 5.54, $\mathrm{N}$ 4.12. MS (ESI ${ }^{+}-\mathrm{TOF}$ in $\mathrm{CH}_{2} \mathrm{Cl}_{2} / \mathrm{MeOH} / \mathrm{NH}_{4} \mathrm{HCOO} 5$ $\mathrm{mM}): m / z=1167.49\left[\mathrm{M}_{2}-\mathrm{AgBr}_{2}\right]^{+}, 637.20[\mathrm{M}-\mathrm{Br}]^{+}, 531.30[2 \mathbf{b}-\mathrm{Br}]^{+}$.

[AgBr\{1-Mes-3-(G2-dend)Imz-2-ylidene\}] (7a): $\operatorname{Ag}_{2} \mathrm{O}(60 \mathrm{mg}, 0.26 \mathrm{mmol})$ and 3a (500 mg, $0.502 \mathrm{mmol})$. Yield: $531 \mathrm{mg}, 96 \% .{ }^{1} \mathrm{H}$ NMR $\left(\mathrm{CDCl}_{3}\right): \delta=7.40-7.26(\mathrm{~m}, 20 \mathrm{H}, \mathrm{Ph}), 7.05\left(\mathrm{~d},{ }^{3} J_{\mathrm{H}, \mathrm{H}}=\right.$ $\left.1.5,1 \mathrm{H}, \mathrm{Imz}-\mathrm{H}^{5}\right), 6.91(\mathrm{~s}, 2 \mathrm{H}, m-\mathrm{Mes}), 6.86\left(\mathrm{~d},{ }^{3} J_{\mathrm{H}, \mathrm{H}}=1.5,1 \mathrm{H}, \mathrm{Imz}-\mathrm{H}^{4}\right), 6.66\left(\mathrm{~d},{ }^{4} J_{\mathrm{H}, \mathrm{H}}=2.2,4 \mathrm{H}\right.$, G1-o-Ar), $6.56\left(\mathrm{t},{ }^{4} J_{\mathrm{H}, \mathrm{H}}=2.2,2 \mathrm{H}, \mathrm{G} 1-p-\mathrm{Ar}\right), 6.54\left(\mathrm{t},{ }^{4} J_{\mathrm{H}, \mathrm{H}}=2.1,1 \mathrm{H}, \mathrm{G} 0-p-\mathrm{Ar}\right), 6.46\left(\mathrm{~d},{ }^{4} J_{\mathrm{H}, \mathrm{H}}=2.1\right.$, 2H, G0-o-Ar), 5.28 (s, 2H, $\mathrm{CH}_{2}$ ), 5.02 (s, 8H, $\left.\mathrm{PhCH}_{2} \mathrm{O}\right), 4.95$ (s, 4H G1-CH $\mathrm{CH}_{2} \mathrm{O}$, 2.30 (s, 3H, Mes$p-M e), 1.95$ ppm (s, 6H, Mes-o-Me). ${ }^{13} \mathrm{C}\left\{{ }^{1} \mathrm{H}\right\}$ NMR $\left(\mathrm{CDCl}_{3}\right): \delta=160.4$ (G0-m-Ar), 160.2 (G1-mAr), 139.5 (para-Mes), 138.9 (G1-ipso-Ar), 137.7 (G0-ipso-Ar), 136.7 (ipso-Ph), 135.2 (ipso-Mes), 134.6 (o-Mes), 129.4 (m-Mes), 128.5 and 127.5 (o- and $m$-Ph), $128.0\left(p\right.$-Ph), 123.0 (Imz-C $\left.{ }^{5}\right), 121.1$ $\left(\mathrm{Imz}-\mathrm{C}^{4}\right), 106.8$ (G0-o-Ar), 106.4 (G1-o-Ar), 102.2 (G0-p-Ar), 101.6 (G1-p-Ar), $70.1\left(\mathrm{PhCH} \mathrm{H}_{2} \mathrm{O}\right)$, $70.0\left(\mathrm{G} 1-\mathrm{CH}_{2} \mathrm{O}\right), 55.7\left(\mathrm{CH}_{2}\right), 21.0$ (Mes- $\left.p-\mathrm{Me}\right), 17.7$ ppm (Mes-o-Me), Imz-C ${ }^{2}$ not observed. IR (KBr pellet): $v=1595$ and 1451 (vs, C-C $\mathrm{C}_{\text {arom}}$ ), 1496 (m, CNC), 1295 (s, C-O-Cas), 1155 and 1052 $\mathrm{cm}^{-1}$ (vs, C-O-C $\mathrm{C}_{\mathrm{s}}$. Elemental analysis (\%) calcd for $\mathrm{C}_{61} \mathrm{H}_{56} \mathrm{AgBrN}_{2} \mathrm{O}_{6}$ (1100.88): C 66.55, H 5.13, 
$\mathrm{N}$ 2.54; found: $\mathrm{C}$ 66.61, $\mathrm{H}$ 5.02, $\mathrm{N}$ 2.68. $\mathrm{MS}\left(\mathrm{ESI}^{+}-\mathrm{TOF}\right.$ in $\left.\mathrm{CH}_{2} \mathrm{Cl}_{2} / \mathrm{MeOH} / \mathrm{NH}_{4} \mathrm{HCOO} 5 \mathrm{mM}\right): m / z=$ $1931.73\left[\mathrm{M}_{2}-\mathrm{AgBr}_{2}\right]^{+}, 913.42[\mathbf{3 a}-\mathrm{Br}]^{+}$.

[AgBr\{1-( $\left.\left.\left.{ }^{i} \mathbf{P r}_{2} \mathbf{P h}\right)-3-(G 2-d e n d) I m z-2-y l i d e n e\right\}\right]$ (7b): $\operatorname{Ag}_{2} \mathrm{O}$ (51 mg, $0.22 \mathrm{mmol}$; plus $10 \mathrm{mg}$, $0.043 \mathrm{mmol}$, added after 3 days) and 3b (450 mg, $0.434 \mathrm{mmol})$. Yield: $452 \mathrm{mg}, 91 \% .{ }^{1} \mathrm{H}$ NMR $\left(\mathrm{CDCl}_{3}\right): \delta=7.30-7.49\left(\mathrm{~m}, 23 \mathrm{H},{ }^{i} \mathrm{Pr}_{2} \mathrm{Ph}\right.$ and $\left.\mathrm{Ph}\right), 7.08\left(\mathrm{t},{ }^{3} J_{\mathrm{H}, \mathrm{H}} \approx{ }^{4} J_{\mathrm{H}, \mathrm{Ag}} \approx 1.7,1 \mathrm{H}, \mathrm{Imz}-\mathrm{H}^{5}\right), 6.95(\mathrm{t}$, $\left.{ }^{3} J_{\mathrm{H}, \mathrm{H}} \approx{ }^{4} J_{\mathrm{H}, \mathrm{Ag}} \approx 1.7,1 \mathrm{H}, \mathrm{Imz}-\mathrm{H}^{4}\right), 6.66\left(\mathrm{~d},{ }^{4} J_{\mathrm{H}, \mathrm{H}}=2.1,4 \mathrm{H}, \mathrm{G} 1-o-\mathrm{Ar}\right), 6.57(\mathrm{t}, 3 \mathrm{H}, \mathrm{G} 0-p-\mathrm{Ar}$ and G1- $p$ Ar), $6.46\left(\mathrm{~d},{ }^{4} J_{\mathrm{H}, \mathrm{H}}=1.7,2 \mathrm{H}, \mathrm{G} 0-o-\mathrm{Ar}\right), 5.32\left(\mathrm{~s}, 2 \mathrm{H}, \mathrm{CH}_{2}\right), 5.02\left(\mathrm{~s}, 8 \mathrm{H}, \mathrm{PhCH}_{2} \mathrm{O}\right), 4.94$ (s, 4H, G1$\left.\mathrm{CH}_{2} \mathrm{O}\right), 2.37\left(\mathrm{sep},{ }^{3} J_{\mathrm{H}, \mathrm{H}}=7.0,2 \mathrm{H}, \mathrm{CHMe}\right), 1.20\left(\mathrm{~d},{ }^{3} J_{\mathrm{H}, \mathrm{H}}=7.0,6 \mathrm{H}, \mathrm{CHMe} e_{2}\right), 1.07\left(\mathrm{~d},{ }^{3} J_{\mathrm{H}, \mathrm{H}}=7.0\right.$, $6 \mathrm{H}, \mathrm{CHMe} 2) .{ }^{13} \mathrm{C}\left\{{ }^{1} \mathrm{H}\right\}$ NMR $\left(\mathrm{CDCl}_{3}\right): \delta=160.4$ (G0-m-Ar), 160.1 (G1-m-Ar), $145.55\left(p-{ }^{i} \mathrm{Pr}_{2} \mathrm{Ph}\right)$, 138.76 (G1-ipso-Ar), 137.6 (G0-ipso-Ar), 136.6 (ipso-Ph), 134.5 (ipso- $\left.{ }^{i} \mathrm{Pr}_{2} \mathrm{Ph}\right), 130.5\left(\right.$ o- $\left.^{i} \mathrm{Pr}_{2} \mathrm{Ph}\right)$, $128.0(p-\mathrm{Ph}), 127.5$ and $128.5(o-$ and $m-\mathrm{Ph}), 124.2\left(\mathrm{Imz}_{-} \mathrm{C}^{5}\right.$ and $m-{ }^{i} \mathrm{Pr}_{2} \mathrm{Ph}$ overlapping), 121.0 $\left({ }^{3} J_{\mathrm{C}, \mathrm{Ag}} \approx 7.0, \mathrm{Imz}_{\mathrm{C}}{ }^{4}\right), 106.6$ (G0-o-Ar), 106.4 (G1-o-Ar), 101.9 (G0-p-Ar), 101.6 (G1-p-Ar), 70.1 ( $\mathrm{Ph}-\mathrm{CH}_{2} \mathrm{O}$ and $\left.\mathrm{Ar}-\mathrm{CH}_{2} \mathrm{O}\right), 55.7\left(\mathrm{CH}_{2}\right), 28.4\left(\mathrm{CHMe}_{2}\right), 24.6(\mathrm{CHMe}), 24.3(\mathrm{CHMe}), \mathrm{Imz}_{2} \mathrm{C}^{2}$ not observed. $\left\{{ }^{1} \mathrm{H},{ }^{13} \mathrm{C}\right\} \mathrm{HMBC}\left(\mathrm{CDCl}_{3}\right): \delta=183.3\left(\mathrm{Imz}-\mathrm{C}^{2},{ }^{1} J_{\mathrm{C}-\mathrm{Ag}} \approx 250\right) . \quad\left\{{ }^{1} \mathrm{H}^{-15} \mathrm{~N}\right\} \mathrm{gHMBC}\left(\mathrm{CDCl}_{3}\right)$ : $\delta=-185.2$ and -185.5. IR (KBr pellet): $v=1595(\mathrm{~m})$ and 1451 (s, C-C arom), 1497 (s, CNC), 1301 $\left(\mathrm{m}, \mathrm{C}-\mathrm{O}-\mathrm{C}_{\mathrm{as}}\right.$ ), 1155 and $1056 \mathrm{~cm}^{-1}$ (vs, C-O-C $\mathrm{C}_{\mathrm{s}}$. Elemental analysis (\%) calcd for $\mathrm{C}_{64} \mathrm{H}_{62} \mathrm{AgBrN}_{2} \mathrm{O}_{6}$ (1142.96): C 67.25, H 5.47, N 2.45; found: C 67.47, H 5.41, N 2.46. MS (ESI ${ }^{+}-\mathrm{TOF}$ in $\left.\mathrm{CH}_{2} \mathrm{Cl}_{2} / \mathrm{MeOH} / \mathrm{NH}_{4} \mathrm{HCOO} 5 \mathrm{mM}\right): m / z=2015.84\left[\mathrm{M}_{2}-\mathrm{AgBr}_{2}\right]^{+}, 1061.38[\mathrm{M}-\mathrm{Br}]^{+}, 955.47[\mathbf{3 b}-$ $\mathrm{Br}]^{+}$.

[AgBr\{1-Mes-3-(G3-dend)Imz-2-ylidene\}] (8a): $\operatorname{Ag}_{2} \mathrm{O}(30 \mathrm{mg}, 0.13 \mathrm{mmol})$ and 4a $(392 \mathrm{mg}$, $0.212 \mathrm{mmol})$. Yield: $369 \mathrm{mg}, 89 \% .{ }^{1} \mathrm{H}$ NMR $\left(\mathrm{CDCl}_{3}\right): \delta=7.40-7.26(\mathrm{~m}, 40 \mathrm{H}, \mathrm{Ph}), 7.03\left(\mathrm{~d},{ }^{3} J_{\mathrm{H}, \mathrm{H}}=\right.$ 1.6, $\left.1 \mathrm{H}, \mathrm{Imz}-\mathrm{H}^{5}\right), 6.88(\mathrm{~s}, 2 \mathrm{H}, m-\mathrm{Mes}), 6.83\left(\mathrm{~d},{ }^{3} J_{\mathrm{H}, \mathrm{H}}=1.6,1 \mathrm{H}, \mathrm{Imz}_{-} \mathrm{H}^{4}\right), 6.65(\mathrm{~m}, 12 \mathrm{H}, \mathrm{G} 1$ and G2$o$-Ar), 6.54 (m, 7H, G0, G1 and G2-p-Ar), $6.46\left(\mathrm{~d},{ }^{4} J_{\mathrm{H}, \mathrm{H}}=1.8,2 \mathrm{H}, \mathrm{G} 0-o-\mathrm{Ar}\right), 5.23$ (s, 2H, $\left.\mathrm{CH}_{2}\right)$, 4.99 (s, 16H, $\mathrm{PhCH}_{2} \mathrm{O}$ ), 4.94 (br s, 12H, G1 and $\mathrm{G} 2-\mathrm{CH}_{2} \mathrm{O}$ ), 2.27 (s, 3H, Mes-p-Me), 1.93 ppm (s, $6 \mathrm{H}, \mathrm{Mes}-\mathrm{o}-\mathrm{Me}) .{ }^{13} \mathrm{C}\left\{{ }^{1} \mathrm{H}\right\} \mathrm{NMR}\left(\mathrm{CDCl}_{3}\right): \delta=160.4$ (G0-m-Ar), 160.1 (G2-m-Ar), 160.0 (G1-m-Ar), 139.5 (para-Mes), 139.2 (G2-ipso-Ar), 138.9 (G1-ipso-Ar), 137.7 (G0-ipso-Ar), 136.7 (ipso-Ph), 135.2 (ipso-Mes), 134.5 (o-Mes), 129.4 (m-Mes), 128.5 and 127.5 (o- and $m$ - $\mathrm{Ph}$ ), 128.0 ( $p$-Ph), $123.0\left(\right.$ Imz-C $\left.^{5}\right), 121.2\left(\right.$ Imz-C $\left.^{4}\right), 106.8$ (G0-o-Ar), 106.4 (G1 and G2-o-Ar), 102.1 (G0-p-Ar), 101.6 (G1-p-Ar), 101.5 (G2-p-Ar), $70.1\left(\mathrm{PhCH}_{2} \mathrm{O}\right.$ and $\left.\mathrm{G} 1-\mathrm{CH}_{2} \mathrm{O}\right), 70.0\left(\mathrm{G} 2-\mathrm{CH}_{2} \mathrm{O}\right), 55.7\left(\mathrm{CH}_{2}\right), 21.0$ (Mes- $p$-Me), 17.7 ppm (Mes-o-Me), Imz-C $\mathrm{C}^{2}$ not observed. IR (KBr pellet): $v=1595$ and 1451 (vs, C-C $\mathrm{C}_{\text {arom}}$ ), 1497 (m, CNC), 1295 (m, C-O-C $\mathrm{C}_{\mathrm{as}}$ ), 1155 and $1052 \mathrm{~cm}^{-1}$ (vs, C-O-C ). Elemental analysis (\%) calcd for $\mathrm{C}_{117} \mathrm{H}_{104} \mathrm{AgBrN}_{2} \mathrm{O}_{14}$ (1949.85): C 72.07, H 5.38, N 1.44; found: C 72.17, H 5.45, N 1.53. $\mathrm{MS}\left(\mathrm{ESI}^{+}-\mathrm{TOF}\right.$ in $\left.\mathrm{CH}_{2} \mathrm{Cl}_{2} / \mathrm{MeOH} / \mathrm{NH}_{4} \mathrm{HCOO} 5 \mathrm{mM}\right): m / z=3628.38\left[\mathrm{M}_{2}-\mathrm{AgBr}_{2}\right]^{+}, 1761.76$ $[\mathbf{4 a}-\mathrm{Br}]^{+}$. 


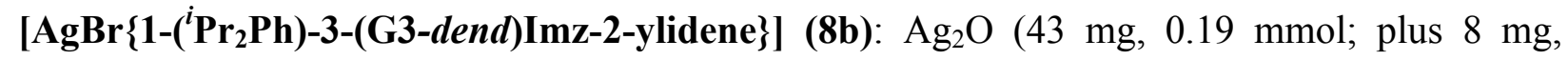
$0.034 \mathrm{mmol}$, added after 5 days) and $\mathbf{4 b}(700 \mathrm{mg}, 0.371 \mathrm{mmol})$. Yield: $500 \mathrm{mg}, 67 \%$. ${ }^{1} \mathrm{H}$ NMR $\left(\mathrm{CDCl}_{3}\right): \delta=7.17-7.39\left(\mathrm{~m}, 43 \mathrm{H},{ }^{i} \mathrm{Pr}_{2} \mathrm{Ph}\right.$ and $\left.\mathrm{Ph}\right), 7.07\left(\mathrm{t},{ }^{3} J_{\mathrm{H}, \mathrm{H}} \approx{ }^{4} J_{\mathrm{H}, \mathrm{Ag}} \approx 1.2,1 \mathrm{H}, \mathrm{Imz}-\mathrm{H}^{5}\right), 6.93(\mathrm{t}$, $\left.{ }^{3} J_{\mathrm{H}, \mathrm{H}} \approx{ }^{4} J_{\mathrm{H}, \mathrm{Ag}} \approx 1.2,1 \mathrm{H}, \mathrm{Imz}-\mathrm{H}^{4}\right), 6.65$ (d, 12H, G1-o-Ar and G2-o-Ar overlapping), 6.54 (t, 7H, G0p-Ar, G1-p-Ar and G2-p-Ar overlapping), $6.46\left(\mathrm{~d},{ }^{4} J_{\mathrm{H}, \mathrm{H}}=2.0,2 \mathrm{H}, \mathrm{G} 0-o-\mathrm{Ar}\right), 5.28\left(\mathrm{~s}, 2 \mathrm{H}, \mathrm{CH}_{2}\right)$, $4.99\left(\mathrm{~s}, 16 \mathrm{H}, \mathrm{PhCH}_{2} \mathrm{O}\right), 4.94$ (s, 12H, G1- $\mathrm{CH}_{2} \mathrm{O}$ and $\mathrm{G} 2-\mathrm{CH}_{2} \mathrm{O}$ overlappig), 2.35 (sep, ${ }^{3} J_{\mathrm{H}, \mathrm{H}}=7.0$, $2 \mathrm{H}, \mathrm{CHMe} 2), 1.20\left(\mathrm{~d},{ }^{3} J_{\mathrm{H}, \mathrm{H}}=7.0,6 \mathrm{H}, \mathrm{CHMe} 2\right), 1.07\left(\mathrm{~d},{ }^{3} J_{\mathrm{H}, \mathrm{H}}=7.0,6 \mathrm{H}, \mathrm{CHMe} e_{2}\right){ }^{13} \mathrm{C}\left\{{ }^{1} \mathrm{H}\right\} \mathrm{NMR}$ $\left(\mathrm{CDCl}_{3}\right): \delta=160.5$ (G0-m-Ar), 160.1 (G2-m-Ar), 160.0 (G1-m-Ar), $145.6\left(p-{ }^{i} \mathrm{Pr}_{2} \mathrm{Ph}\right), 139.2$ (G2ipso-Ar), 138.8 (G1-ipso-Ar), 137.8 (G0-ipso-Ar), 136.7 (ipso-Ph), 134.5 (ipso- $\left.{ }^{i} \mathrm{Pr}_{2} \mathrm{Ph}\right), 130.6$ (o$\left.{ }^{i} \mathrm{Pr}_{2} \mathrm{Ph}\right), 128.0(p-\mathrm{Ph}), 127.5$ and 128.5 (o- and $\left.m-\mathrm{Ph}\right), 124.7$ (Imz-C ${ }^{5}$ and $m-\mathrm{C}_{6} \mathrm{H}_{3}{ }^{i} \mathrm{Pr}_{2}$ overlapping), $121.0\left({ }^{3} J_{\mathrm{C}, \mathrm{Ag}} \approx 6.5, \mathrm{Imz}^{-C^{4}}\right), 106.5$ (G0-o-Ar), 106.5 (G1-o-Ar), 106.5 (G2-o-Ar), 102.0 (G0-p-Ar), 101.5 (G1-p-Ar and G2-p-Ar overlapping), 70.1 ( $\mathrm{Ph}-\mathrm{CH}_{2} \mathrm{O}$ and $\mathrm{G} 2-\mathrm{Ar}-\mathrm{CH}_{2} \mathrm{O}$ overlapping), 70.0 (G1-Ar- $\left.\mathrm{CH}_{2} \mathrm{O}\right), 55.7\left(\mathrm{CH}_{2}\right), 28.3\left(\mathrm{CHMe}_{2}\right), 24.6\left(\mathrm{CHMe}_{2}\right), 24.3(\mathrm{CHMe})$, Imz-C not observed. IR (KBr pellet): $v=1595$ (m) and 1451 (s, C-C arom), 1497 (s, CNC), 1295 (m, C-O-C $\mathrm{C}_{\mathrm{as}}$ ), 1155 and 1054 $\mathrm{cm}^{-1}$ (vs, C-O-C $\mathrm{s}_{\mathrm{s}}$. Elemental analysis (\%) calcd for $\mathrm{C}_{120} \mathrm{H}_{110} \mathrm{AgBrN}_{2} \mathrm{O}_{14}$ (1991.93): C 72.36, H 5.57, $\mathrm{N} 1.41$; found: $\mathrm{C} 72.50, \mathrm{H}$ 5.89, $\mathrm{N}$ 1.57. $\mathrm{MS}$ (ESI ${ }^{+}-\mathrm{TOF}$ in $\mathrm{CH}_{2} \mathrm{Cl}_{2} / \mathrm{MeOH} / \mathrm{NH}_{4} \mathrm{HCOO} 5$ $\mathrm{mM}): m / z=3712.50\left[\mathrm{M}_{2}-\mathrm{AgBr}_{2}\right]^{+}, 1909.73[\mathrm{M}-\mathrm{Br}]^{+}, 1803.80[\mathbf{4 b}-\mathrm{Br}]^{+}$. 


\section{Preparation and characterization data for NHC-Pd complexes $9 a-12 a$ and $9 b-12 b$}

$\left[\mathrm{PdBr}_{2}(\mathrm{cod})\right]$ was added to an Schlenk tube containing a $\mathrm{CH}_{2} \mathrm{Cl}_{2}(20-30 \mathrm{~mL})$ solution of the corresponding silver compound $\mathbf{5 a}-\mathbf{8 a}$ or $\mathbf{5 b}-\mathbf{8 b}$. The reaction was stirred at room temperature $1 \mathrm{~h}$ for $9 \mathrm{a}$ and $\mathbf{1 0 a}$, or $2 \mathrm{~h}$ for $\mathbf{1 1 a}$ and $\mathbf{1 2 a}$, whereas $3,6,9$, and $12 \mathrm{~h}$ were necessary for completion of the formation of $\mathbf{9 b}, \mathbf{1 0 b}, \mathbf{1 1 b}$, and $\mathbf{1 2 b}$, respectively. The resulting solution was filtered and evaporated to dryness under vacuum, and the residue washed with hexane for $\mathbf{9 a - 1 2 a}$, or washed in solution of $\mathrm{CH}_{2} \mathrm{Cl}_{2}(20 \mathrm{~mL})$ with water $(3 \times 15 \mathrm{~mL})$ for $\mathbf{9 b}-\mathbf{1 2 b}$, for which the organic layer was subsequently dried over $\mathrm{MgSO}_{4}$. After evaporation of the solvent, the bis(NHC) complexes were isolated as white to pale yellow solids (orange in the case of 12a). Complexes $\mathbf{1 1 b}$ and $\mathbf{1 2 b}$ were additionally purified by column chromatography over silica gel using ethyl acetate/hexane (1:1) as the eluent. In solution, the palladium complexes consisted in a mixture of trans-syn and trans-anti isomers (ca. 65/35, 70/30, and 75/25\% for 9a-12a, 9b-10b, and 11b-12b, respectively).

[PdBr 2 1-Mes-3-(G0-dend)Imz-2-ylidene $\left.\}_{2}\right]$ (9a): $\left[\mathrm{PdBr}_{2}(\mathrm{COD})\right]$ (160 mg, $\left.0.427 \mathrm{mmol}\right)$ and 5a (397 mg, $0.855 \mathrm{mmol})$. Yield: $322 \mathrm{mg}, 92 \%$. trans-syn 9a: ${ }^{1} \mathrm{H} \mathrm{NMR}\left(\mathrm{CDCl}_{3}\right): \delta=7.48-7.26(\mathrm{~m}$, $10 \mathrm{H}, \mathrm{Ph}), 6.85(\mathrm{~s}, 4 \mathrm{H}, m-\mathrm{Mes}), 6.69\left(\mathrm{~d},{ }^{3} J_{\mathrm{H}, \mathrm{H}}=1.8,2 \mathrm{H}, \mathrm{Imz}_{-} \mathrm{H}^{5}\right), 6.64\left(\mathrm{~d},{ }^{3} J_{\mathrm{H}, \mathrm{H}}=1.8,2 \mathrm{H}, \mathrm{Imz}-\mathrm{H}^{4}\right)$, $5.77\left(\mathrm{~s}, 4 \mathrm{H}, \mathrm{CH}_{2}\right), 2.46$ (s, 6H, Mes-p-Me), $1.98 \mathrm{ppm}(\mathrm{s}, 12 \mathrm{H}, \mathrm{Mes}-o-M e),{ }^{13} \mathrm{C}\left\{{ }^{1} \mathrm{H}\right\}$ NMR $\left(\mathrm{CDCl}_{3}\right)$ : $\delta=170.0$ (Imz-C ${ }^{2}$ ), 137.6 (p-Mes), 136.4 (ipso-Ph), 136.0 (o-Mes), 135.4 (ipso-Mes), 129.0 (mMes), 128.9 and 128.7 (o- and $m-\mathrm{Ph}), 128.0(p-\mathrm{Ph}), 123.5\left(\mathrm{Imz}^{-} \mathrm{C}^{5}\right), 120.4\left(\mathrm{Imz}^{-} \mathrm{C}^{4}\right), 55.1\left(\mathrm{CH}_{2}\right)$, $21.2\left(\right.$ Mes-p-Me), 19.4 ppm (Mes-o-Me). trans-anti 9a: ${ }^{1} \mathrm{H}$ NMR $\left(\mathrm{CDCl}_{3}\right): \delta=7.48-7.26(\mathrm{~m}, 10 \mathrm{H}$, $\mathrm{Ph}), 6.76(\mathrm{~s}, 4 \mathrm{H}, m-\mathrm{Mes}), 6.74\left(\mathrm{~d},{ }^{3} J_{\mathrm{H}, \mathrm{H}}=1.8 \mathrm{~Hz}, 2 \mathrm{H}, \mathrm{Imz}_{-} \mathrm{H}^{5}\right), 6.70\left(\mathrm{~d},{ }^{3} J_{\mathrm{H}, \mathrm{H}}=1.8 \mathrm{~Hz}, 2 \mathrm{H}, \mathrm{Imz}_{-} \mathrm{H}^{4}\right)$, 5.45 (s, 4H, $\left.\mathrm{CH}_{2}\right), 2.20$ (s, 12H, Mes-o-Me), $2.11 \mathrm{ppm}(\mathrm{s}, 6 \mathrm{H}, \mathrm{Mes}-p-M e) .{ }^{13} \mathrm{C}\left\{{ }^{1} \mathrm{H}\right\} \mathrm{NMR}\left(\mathrm{CDCl}_{3}\right)$ : $\delta=170.1$ (Imz-C ${ }^{2}$ ), 138.3 (p-Mes), 136.3 (ipso-Ph), 136.0 (o-Mes), 135.6 (ipso-Mes), 128.8 (mMes), 128.9 and $128.6(o-$ and $m-\mathrm{Ph}), 127.9(p-\mathrm{Ph}), 123.5\left(\mathrm{Imz}^{-} \mathrm{C}^{5}\right), 120.2\left(\mathrm{Imz}^{-}{ }^{4}\right), 54.8\left(\mathrm{CH}_{2}\right)$, 20.9 (Mes-p-Me), 19.8 ppm (Mes-o-Me). cis-anti 9: ${ }^{1} \mathrm{H}$ NMR $\left(\mathrm{CDCl}_{3}\right): \delta=7.40-7.31$ (m, 10H, $\mathrm{Ph}$ ), $7.11(\mathrm{~s}, 2 \mathrm{H}, m-\mathrm{Mes}), 7.06(\mathrm{~s}, 2 \mathrm{H}, m-\mathrm{Mes}), 6.68\left(\mathrm{~d},{ }^{3} J_{\mathrm{H}, \mathrm{H}}=1.8 \mathrm{~Hz}, 2 \mathrm{H}, \mathrm{Imz}-\mathrm{H}^{5}\right), 6.58\left(\mathrm{~d},{ }^{3} J_{\mathrm{H}, \mathrm{H}}=1.8\right.$ $\left.\mathrm{Hz}, 2 \mathrm{H}, \mathrm{Imz}_{-} \mathrm{H}^{4}\right), 6.41\left(\mathrm{~d}, J_{\mathrm{H}, \mathrm{H}}=14.1 \mathrm{~Hz}, 1 \mathrm{H}, \mathrm{CH}_{2}\right), 3.35\left(\mathrm{~d}, J_{\mathrm{H}, \mathrm{H}}=14.1 \mathrm{~Hz}, 1 \mathrm{H}, \mathrm{CH}_{2}\right), 2.42(\mathrm{~s}, 6 \mathrm{H}$, Mes-o-Me), 2.41 (s, 6H, Mes-o-Me), 1.58 ppm (s, 6H, Mes- $p-M e) .{ }^{13} \mathrm{C}\left\{{ }^{1} \mathrm{H}\right\} \mathrm{NMR}\left(\mathrm{CDCl}_{3}\right): \delta=$ 163.7 (Imz-C ${ }^{2}$ ), 139.9 (p-Mes), 135.4 and 139.5 (o-Mes), 134.8 (ipso-Ph), 133.9 (ipso-Mes), 130.0 and $128.8(o-$ and $m-\mathrm{Ph}), 128.7(p-\mathrm{Ph}), 128.5$ and $131.0\left(m\right.$-Mes), $123.1\left(\mathrm{Imz}^{-} \mathrm{C}^{5}\right), 120.9\left(\mathrm{Imz}-\mathrm{C}^{4}\right)$, $53.7\left(\mathrm{CH}_{2}\right), 18.2,20.8$ and 21.0 ppm (Mes-Me). IR (KBr pellet): $v=1606(\mathrm{~m})$ and $1450(\mathrm{~s}, \mathrm{C}-$ $\mathrm{C}_{\text {arom }}$ ), $1487 \mathrm{~cm}^{-1}$ (s, CNC). Elemental analysis (\%) calcd for $\mathrm{C}_{38} \mathrm{H}_{40} \mathrm{Br}_{2} \mathrm{~N}_{4} \mathrm{Pd}$ (818.98): C 55.73, $\mathrm{H}$ 4.92, N 6.84; found: $\mathrm{C} 55.16, \mathrm{H} 4.74, \mathrm{~N}$ 6.95. $\mathrm{MS}$ (ESI ${ }^{+}$TOF in $\left.\mathrm{CH}_{2} \mathrm{Cl}_{2} / \mathrm{MeOH} / \mathrm{NH}_{4} \mathrm{HCOO} 5 \mathrm{mM}\right)$ : $m / z=834.10\left[\mathrm{M}+\mathrm{NH}_{4}\right]^{+}, 737.15[\mathrm{M}-\mathrm{Br}]^{+}, 277.17[\mathbf{1 a}-\mathrm{Br}]^{+}$. 


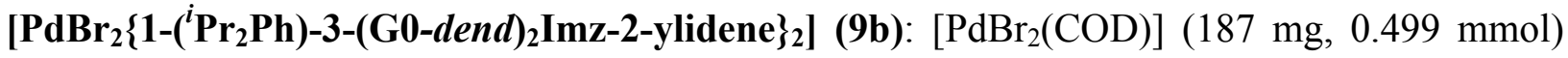
and 5b (506 mg, $0.998 \mathrm{mmol})$. Yield: $689 \mathrm{mg}$, 93\%. trans-syn-9b: ${ }^{1} \mathrm{H} \mathrm{NMR}\left(\mathrm{CDCl}_{3}\right): \delta=7.40-$ 7.51(m, 4H, $o-\mathrm{Ph}), 7.19-7.36\left(\mathrm{~m}, 6 \mathrm{H}, m\right.$ - and $p-\mathrm{Ph}$ ), 7.08 (broad s, 4H, $m-{ }_{-}^{i} \mathrm{Pr}_{2} \mathrm{Ph}$ ), 7.04 (broad s, 2H, $p$ - ${ }^{i} \mathrm{Pr}_{2} \mathrm{Ph}$ ), 6.67 (broad s, 2H, Imz-H ${ }^{5}$ ), 6.42 (broad s, 2H, Imz-H ${ }^{4}$ ), 5.96 (s, 4H, CH ), 2.77 (sep, $\left.{ }^{3} J_{H, H}=6.9,4 \mathrm{H}, \mathrm{CHMe}\right), 0.95\left(\mathrm{~d},{ }^{3} J_{H, H}=6.9,12 \mathrm{H}, \mathrm{CHMe} 2\right), 0.89\left(\mathrm{~d},{ }^{3} J_{H, H}=6.9,12 \mathrm{H}, \mathrm{CHMe} e_{2}\right)$. ${ }^{13} \mathrm{C}\left\{{ }^{1} \mathrm{H}\right\} \mathrm{NMR}\left(\mathrm{CDCl}_{3}\right): \delta=170.5\left(\mathrm{Imz} \mathrm{C}^{2}\right), 146.5\left(p-{ }^{i} \mathrm{Pr}_{2} \mathrm{Ph}\right), 135.9$ (ipso- $\left.{ }^{i} \mathrm{Pr}_{2} \mathrm{Ph}\right), 135.9$ (ipso-Ph), $129.6\left(o^{-}{ }^{i} \mathrm{Pr}_{2} \mathrm{Ph}\right), 129.4(o-\mathrm{Ph}), 128.8(p-\mathrm{Ph}), 128.1(\mathrm{~m}-\mathrm{Ph}), 124.0\left(\mathrm{Imz}-\mathrm{C}^{5}\right), 123.7\left(\mathrm{~m}^{i}{ }^{i} \mathrm{Pr}_{2} \mathrm{Ph}\right), 119.4$ $\left(\mathrm{Imz}^{\mathrm{C}} \mathrm{C}^{4}\right), 55.1\left(\mathrm{CH}_{2}\right), 28.1\left(\mathrm{CHMe}_{2}\right), 26.4\left(\mathrm{CHMe}_{2}\right), 23.0(\mathrm{CHMe})$. trans-anti-9b: ${ }^{1} \mathrm{H}$ NMR $\left(\mathrm{CDCl}_{3}\right): \delta=7.40-7.51(\mathrm{~m}, 4 \mathrm{H}, o-\mathrm{Ph}), 7.19-7.36(\mathrm{~m}, 6 \mathrm{H}, m$ - and $p-\mathrm{Ph}), 7.08$ (broad s, 4H, $m-$ ${ }^{i} \mathrm{Pr}_{2} \mathrm{Ph}$ ), 7.04 (broad s, 2H, $p-{ }^{i} \mathrm{Pr}_{2} \mathrm{Ph}$ ), 6.77 (broad s, 2H, Imz-H ${ }^{5}$ ), 6.68 (broad s, 2H, Imz-H ${ }^{4}$ ), 5.42 (s, 4H, $\left.\mathrm{CH}_{2}\right), 3.05\left(\mathrm{sep},{ }^{3} J_{H, H}=6.9,4 \mathrm{H},\left(\mathrm{CHMe}_{2}\right), 1.28\left(\mathrm{~d},{ }^{3} J_{H, H}=6.9,12 \mathrm{H},(\mathrm{CHMe}), 0.99\left(\mathrm{~d},{ }^{3} J_{H, H}\right.\right.\right.$ $=6.9,12 \mathrm{H},(\mathrm{CHMe}) \cdot{ }^{13} \mathrm{C}\left\{{ }^{1} \mathrm{H}\right\} \mathrm{NMR}\left(\mathrm{CDCl}_{3}\right): \delta=171.1\left(\mathrm{Imz}_{-} \mathrm{C}^{2}\right), 147.1\left(p-{ }^{i} \mathrm{Pr}_{2} \mathrm{Ph}\right), 135.3$ (ipso$\left.{ }^{i} \mathrm{Pr}_{2} \mathrm{Ph}\right), 135.0$ (ipso-Ph), $129.3\left(o^{i}{ }^{i} \mathrm{Pr}_{2} \mathrm{Ph}\right), 129.2(o-\mathrm{Ph}), 128.6$ (p-Ph), 127.9 (m-Ph), 124.7 (Imz$\left.\mathrm{C}^{5}\right), 123.3\left(m{ }^{i}{ }^{-}{ }_{2} \mathrm{Ph}\right), 119.2\left(\mathrm{Imz} \mathrm{C}^{4}\right), 54.7\left(\mathrm{CH}_{2}\right), 28.5\left(\mathrm{CHMe}_{2}\right), 26.5(\mathrm{CHMe}), 22.8(\mathrm{CHMe}) . \mathrm{IR}$ (KBr pellet): $v=1591$ and 1457 (s, C-C $\left.\mathrm{C}_{\mathrm{arom}}\right), 1497 \mathrm{~cm}^{-1}$ (s, CNC). Elemental analysis (\%) calcd for $\mathrm{C}_{44} \mathrm{H}_{52} \mathrm{Br}_{2} \mathrm{~N}_{4} \mathrm{Pd}$ (903.14): C 58.51, H 5.80, N 6.20; found: C 58.49, H 5.94, N 6.14. MS (ESI ${ }^{+}-\mathrm{TOF}$ in $\left.\mathrm{CH}_{2} \mathrm{Cl}_{2} / \mathrm{MeOH} / \mathrm{NH}_{4} \mathrm{HCOO} 5 \mathrm{mM}\right): m / z=918.20\left[\mathrm{M}+\mathrm{NH}_{4}\right]^{+}, 821.25[\mathrm{M}-\mathrm{Br}]^{+}, 319.22[\mathbf{1 b}-$ $\mathrm{Br}]^{+}$.

[PdBr 2 \{1-Mes-3-(G1-dend)Imz-2-ylidene $\left.\}_{2}\right]$ (10a): [PdBr $\left.2(\mathrm{COD})\right]$ (69 mg, $\left.0.184 \mathrm{mmol}\right)$ and 6a (0.250 g, $0.369 \mathrm{mmol})$. Yield: $216 \mathrm{mg}, 94 \%$. trans-syn 10a: ${ }^{1} \mathrm{H} \mathrm{NMR}\left(\mathrm{CDCl}_{3}\right): \delta=7.45-7.26(\mathrm{~m}$, $20 \mathrm{H}, \mathrm{Ph}), 6.85(\mathrm{~s}, 4 \mathrm{H}, m-\mathrm{Mes}), 6.78\left(\mathrm{~d},{ }^{3} J_{\mathrm{H}, \mathrm{H}}=1.8 \mathrm{~Hz}, 2 \mathrm{H}, \mathrm{Imz}_{-} \mathrm{H}^{5}\right), 6.75\left(\mathrm{~d},{ }^{3} J_{\mathrm{H}, \mathrm{H}}=1.8 \mathrm{~Hz}, 2 \mathrm{H}\right.$, $\left.\operatorname{Imz}-\mathrm{H}^{4}\right), 6.65\left(\mathrm{~d},{ }^{4} J_{\mathrm{H}, \mathrm{H}}=2.2 \mathrm{~Hz}, 4 \mathrm{H}, o-\mathrm{Ar}\right), 6.50\left(\mathrm{t},{ }^{4} J_{\mathrm{H}, \mathrm{H}}=2.2 \mathrm{~Hz}, 2 \mathrm{H}, p-\mathrm{Ar}\right), 5.74\left(\mathrm{~s}, 4 \mathrm{H}, \mathrm{CH}_{2}\right)$, $4.92\left(\mathrm{~s}, 8 \mathrm{H}, \mathrm{PhCH}_{2} \mathrm{O}\right), 2.46$ (s, 6H, Mes- $\left.-\mathrm{Me}\right), 1.98$ ppm (s, 12H, Mes-o-Me). ${ }^{13} \mathrm{C}\left\{{ }^{1} \mathrm{H}\right\} \mathrm{NMR}$ $\left(\mathrm{CDCl}_{3}\right): \delta=170.0$ (Imz-C $\left.{ }^{2}\right), 160.2$ (m-Ar), 138.9 (ipso-Ar), 137.6 (p-Mes), 136.9 (ipso-Ph), 135.9 (o-Mes), 135.4 (ipso-Mes), 128.9 (m-Mes), 128.4 and 127.6 (o- and $m$-Ph), 127.8 ( $p$-Ph), 123.7 $\left(\mathrm{Imz}-\mathrm{C}^{5}\right), 120.6\left(\mathrm{Imz}^{4}{ }^{4}\right), 102.3$ (p-Ar), 107.5 (o-Ar), $70.2\left(\mathrm{PhCH}_{2} \mathrm{O}\right), 55.1\left(\mathrm{CH}_{2}\right), 21.2(\mathrm{Mes}-p$ Me), 19.3 ppm (Mes-o-Me). trans-anti 10a: ${ }^{1} \mathrm{H} \mathrm{NMR}\left(\mathrm{CDCl}_{3}\right): \delta=7.45-7.26(\mathrm{~m}, 20 \mathrm{H}, \mathrm{Ph}), 6.79(\mathrm{~s}$ overlapped, 4H, m-Mes), $6.71\left(\mathrm{~d},{ }^{3} J_{\mathrm{H}, \mathrm{H}}=1.8 \mathrm{~Hz}, 2 \mathrm{H}, \mathrm{Imz}_{-} \mathrm{H}^{5}\right), 6.68\left(\mathrm{~d},{ }^{4} J_{\mathrm{H}, \mathrm{H}}=2.2 \mathrm{~Hz}, 4 \mathrm{H}, o-\mathrm{Ar}\right)$, 6.65 (d overlapped, 2H, Imz-H $\left.{ }^{4}\right), 6.59\left(\mathrm{t},{ }^{4} J_{\mathrm{H}, \mathrm{H}}=2.2 \mathrm{~Hz}, 2 \mathrm{H}, p-\mathrm{Ar}\right), 5.48\left(\mathrm{~s}, 4 \mathrm{H}, \mathrm{CH}_{2}\right), 4.97(\mathrm{~s}, 8 \mathrm{H}$, $\left.\mathrm{PhCH}_{2} \mathrm{O}\right), 2.19\left(\mathrm{~s}, 12 \mathrm{H}\right.$, Mes-o-Me), $2.06 \mathrm{ppm}(\mathrm{s}, 6 \mathrm{H}, \mathrm{Mes}-p-M e) .{ }^{13} \mathrm{C}\left\{{ }^{1} \mathrm{H}\right\} \mathrm{NMR}\left(\mathrm{CDCl}_{3}\right): \delta=$ 170.0 (Imz-C ${ }^{2}$ ), 160.0 (m-Ar), 139.1 (ipso-Ar), 138.5 (p-Mes), 137.2 (ipso-Ph), 136.0 (o-Mes), 135.6 (ipso-Mes), 128.6 ( $m$-Mes), 127.7 (p-Ph), 128.5 and 127.3 (o- and $m$-Ph), 123.9 (Imz-C ${ }^{5}$ ), 120.4 ( $\mathrm{Imz}^{-\mathrm{C}^{4}}$ ), 107.1 (o-Ar), 102.1 (p-Ar), $70.4\left(\mathrm{PhCH}_{2} \mathrm{O}\right), 54.9\left(\mathrm{CH}_{2}\right), 20.8$ (Mes-p-Me), 19.8 ppm (Mes-o-Me). IR (KBr pellet): $v=1592$ and 1449 (vs, C-C $\mathrm{C}_{\text {arom }}$ ), 1496 (m, CNC), 1297 (m, C-O$\mathrm{C}_{\mathrm{as}}$ ), 1162 and $1058 \mathrm{~cm}^{-1}$ (vs, C-O-C ). Elemental analysis (\%) calcd for $\mathrm{C}_{66} \mathrm{H}_{64} \mathrm{Br}_{2} \mathrm{~N}_{4} \mathrm{O}_{4} \mathrm{Pd}$ 
(1243.47): $\mathrm{C}$ 63.75, $\mathrm{H}$ 5.19, $\mathrm{N}$ 4.51; found: $\mathrm{C}$ 63.28, $\mathrm{H}$ 5.25, $\mathrm{N}$ 4.59. MS (ESI ${ }^{+} \mathrm{TOF}$ in $\left.\mathrm{CH}_{2} \mathrm{Cl}_{2} / \mathrm{MeOH} / \mathrm{NH}_{4} \mathrm{HCOO} 5 \mathrm{mM}\right): m / z=1258.27\left[\mathrm{M}+\mathrm{NH}_{4}\right]^{+}, 1161.32[\mathrm{M}-\mathrm{Br}]^{+}$.

[PdBr 2 \{1-( $\left.{ }^{i} \operatorname{Pr}_{2} \mathbf{P h}\right)-3-(G 1-d e n d)_{2}$ Imz-2-ylidene $\left.\}_{2}\right]$ (10b): [PdBr $\left.2(\mathrm{COD})\right]$ (104 mg, 0.278 mmol) and $\mathbf{6 b}$ (400 mg, $0.557 \mathrm{mmol})$. Yield: $335 \mathrm{mg}, 91 \%$. trans-syn-10b: ${ }^{1} \mathrm{H}$ NMR $\left(\mathrm{CDCl}_{3}\right): \delta=7.29$ $7.44(\mathrm{~m}, 20 \mathrm{H}, \mathrm{Ph}), 7.08$ (broad s, $\left.4 \mathrm{H}, m-{ }^{i} \mathrm{Pr} 2 \mathrm{Ph}\right), 7.04$ (broad s, $\left.2 \mathrm{H}, p-{ }^{i} \mathrm{Pr}_{2} \mathrm{Ph}\right), 6.81\left(\mathrm{~d},{ }^{4} J_{H, H}=2.1\right.$, $4 \mathrm{H}, o-\mathrm{Ar}), 6.76\left(\right.$ broad s, 2H, Imz-H $\left.\mathrm{H}^{5}\right), 6.60$ (broad s, 2H, Imz-H $\left.\mathrm{H}^{4}\right), 6.51\left(\mathrm{t},{ }^{4} J_{H, H}=2.1,2 \mathrm{H}, p-\mathrm{Ar}\right)$, $5.87\left(\mathrm{~s}, 4 \mathrm{H}, \mathrm{CH}_{2}\right), 4.91\left(\mathrm{~s}, 8 \mathrm{H}, \mathrm{PhCH}_{2} \mathrm{O}\right), 2.78\left(\mathrm{sep},{ }^{3} J_{H, H}=6.8,4 \mathrm{H}, \mathrm{CHMe}\right), 0.91\left(\mathrm{~d},{ }^{3} J_{H, H}=6.8\right.$, $12 \mathrm{H}, \mathrm{CHMe} 2), 0.87\left(\mathrm{~d},{ }^{3} J_{H, H}=6.8,12 \mathrm{H}, \mathrm{CHMe} 2\right) .{ }^{13} \mathrm{C}\left\{{ }^{1} \mathrm{H}\right\} \mathrm{NMR}\left(\mathrm{CDCl}_{3}\right): \delta=171.6\left(\mathrm{Imz}_{-} \mathrm{C}^{2}\right)$, 160.1 (G0-m-Ar), 146.5 ( $p$ - $\left.{ }^{i} \mathrm{Pr}_{2} \mathrm{Ph}\right), 139.9$ (ipso-Ar), 136.9 (ipso-Ph), 135.1 (ipso- $\left.{ }^{i} \mathrm{Pr}_{2} \mathrm{Ph}\right), 129.5$ (o$\left.{ }^{i} \mathrm{Pr}_{2} \mathrm{Ph}\right), 128.4(o-\mathrm{Ph}), 127.9(p-\mathrm{Ph}), 127.8(m-\mathrm{Ph}), 125.2\left(\mathrm{Imz} \mathrm{C}^{5}\right), 123.6\left(m^{-}{ }^{i} \mathrm{Pr}_{2} \mathrm{Ph}\right), 119.9(\mathrm{Imz}-$ $\left.\mathrm{C}^{4}\right), 107.3$ (G0-o-Ar), 102.2 (G0-p-Ar), $70.1\left(\mathrm{Ph}-\mathrm{CH}_{2} \mathrm{O}\right), 55.1\left(\mathrm{CH}_{2}\right), 28.2\left(\mathrm{CHMe}_{2}\right), 26.3\left(\mathrm{CHMe}_{2}\right)$, $23.0\left(\mathrm{CHMe}_{2}\right)$. trans-anti-10b: ${ }^{1} \mathrm{H} \mathrm{NMR}\left(\mathrm{CDCl}_{3}\right): \delta=7.29-7.44(\mathrm{~m}, 20 \mathrm{H}, \mathrm{Ph}), 7.05$ (broad s, $4 \mathrm{H}$, $m-{ }^{i}{ }^{P} r_{2} \mathrm{Ph}$ ), 7.01 (broad s, 2H, $\left.p-{ }^{i} \mathrm{Pr}_{2} \mathrm{Ph}\right), 6.81$ (broad s, 2H, Imz-H ${ }^{5}$ ), 6.76 (broad s, 2H, Imz-H ${ }^{4}$ ), $6.71\left(\mathrm{~d},{ }^{4} J_{H, H}=2.1,4 \mathrm{H}, o-\mathrm{Ar}\right), 6.56\left(\mathrm{t},{ }^{4} J_{H, H}=1.8,2 \mathrm{H}, p-\mathrm{Ar}\right), 5.44\left(\mathrm{~s}, 4 \mathrm{H}, \mathrm{CH}_{2}\right), 4.95(\mathrm{~s}, 8 \mathrm{H}$, $\left.\mathrm{PhCH}_{2} \mathrm{O}\right), 3.03\left(\mathrm{sep},{ }^{3} J_{H, H}=6.8,4 \mathrm{H}, \mathrm{CHMe}\right), 1.19\left(\mathrm{~d},{ }^{3} J_{H, H}=6.8,12 \mathrm{H}, \mathrm{CHMe}\right), 0.88\left(\mathrm{~d},{ }^{3} J_{H, H}=\right.$ 6.8, 12H, CHMe 2$).{ }^{13} \mathrm{C}\left\{{ }^{1} \mathrm{H}\right\}$ NMR $\left(\mathrm{CDCl}_{3}\right): \delta=171.0\left(\mathrm{Imz}^{2} \mathrm{C}^{2}\right), 160.0$ (G0-m-Ar), $146.7\left(p-{ }^{i} \mathrm{Pr}_{2} \mathrm{Ph}\right)$, 138.7 (ipso-Ar), 136.8 (ipso-Ph), 135.0 (ipso- $\left.{ }^{i}{ }_{2}{ }_{2} \mathrm{Ph}\right), 129.6\left(o-{ }^{i} \mathrm{Pr}_{2} \mathrm{Ph}\right), 128.5$ (o-Ph), 127.8 (p-Ph), $127.6(m-\mathrm{Ph}), 125.6\left(\mathrm{Imz}^{-} \mathrm{C}^{5}\right), 123.1\left(m^{i}{ }^{\mathrm{P}} \mathrm{r}_{2} \mathrm{Ph}\right), 119.5$ (Imz-C $\left.{ }^{4}\right), 107.1$ (G0-o-Ar), 102.1 (G0-p-Ar), $\left.70.1\left(\mathrm{Ph}-\mathrm{CH}_{2} \mathrm{O}\right), 54.8\left(\mathrm{CH}_{2}\right), 28.5\left(\mathrm{CHMe}_{2}\right), 26.6(\mathrm{CHMe}), 22.6(\mathrm{CHMe})_{2}\right)$ IR $(\mathrm{KBr}$ pellet $): v=$ $1596(\mathrm{~m})$ and 1451 (s, C-C arom), 1496 (s, CNC), $1292\left(\mathrm{~m}, \mathrm{C}-\mathrm{O}-\mathrm{C}_{\mathrm{as}}\right), 1151$ and $1051 \mathrm{~cm}^{-1}$ (s, C-O$\mathrm{C}_{\mathrm{s}}$ ). Elemental analysis (\%) calcd for $\mathrm{C}_{72} \mathrm{H}_{76} \mathrm{Br}_{2} \mathrm{~N}_{4} \mathrm{O}_{4} \mathrm{Pd}$ (1327.63): C 65.14, H 5.77, N 4.22; Found (\%): C 65.07, H 5.93, N 4.14. MS (ESI ${ }^{+}-\mathrm{TOF}$ in $\left.\mathrm{CH}_{2} \mathrm{Cl}_{2} / \mathrm{MeOH} / \mathrm{NH}_{4} \mathrm{HCOO} 5 \mathrm{mM}\right): \mathrm{m} / z=1342.36$ $\left[\mathrm{M}+\mathrm{NH}_{4}\right]^{+}, 1245.40[\mathrm{M}-\mathrm{Br}]^{+}, 531.30[2 \mathbf{b}-\mathrm{Br}]^{+}$.

[PdBr 2 \{1-Mes-3-(G2-dend)Imz-2-ylidene $\left.\}_{2}\right]$ (11a): [PdBr $\left.(\mathrm{COD})\right]$ (55 mg, $0.147 \mathrm{mmol}$ ) and 7a (326 mg, 0.296 mmol). Yield: 279 mg, 91\%. trans-syn 11a: ${ }^{1} \mathrm{H}$ NMR $\left(\mathrm{CDCl}_{3}\right): \delta=7.40-7.425(\mathrm{~m}$, $40 \mathrm{H}, \mathrm{Ph}), 6.84$ (s, 4H, m-Mes), 6.71-6.61 (m, 16H, Imz, and G0- and G1-o-Ar), 6.54 (t, ${ }^{4} J_{\mathrm{H}, \mathrm{H}}=2.2$ $\mathrm{Hz}, 2 \mathrm{H}, \mathrm{G} 0-p-\mathrm{Ar}), 6.50\left(\mathrm{t},{ }^{4} \mathrm{~J}_{\mathrm{H}, \mathrm{H}}=2.2 \mathrm{~Hz}, 4 \mathrm{H}, \mathrm{G} 1-p-\mathrm{Ar}\right), 5.65\left(\mathrm{~s}, 4 \mathrm{H}, \mathrm{CH}_{2}\right), 4.95\left(\mathrm{~s}, 16 \mathrm{H}, \mathrm{PhCH}_{2} \mathrm{O}\right)$, $4.82\left(\mathrm{~s}, 8 \mathrm{H}, \mathrm{G} 1-\mathrm{CH}_{2} \mathrm{O}\right), 2.46(\mathrm{~s}, 6 \mathrm{H}, \mathrm{Mes}-p-\mathrm{Me}), 1.98 \mathrm{ppm}(\mathrm{s}, 12 \mathrm{H}, \mathrm{Mes}-\mathrm{o}-\mathrm{Me}) .{ }^{13} \mathrm{C}\left\{{ }^{1} \mathrm{H}\right\} \mathrm{NMR}$ $\left(\mathrm{CDCl}_{3}\right): \delta=170.1$ (Imz-C $\left.{ }^{2}\right), 160.1$ (G0-m-Ar), 160.0 (G1-m-Ar), 139.4 (G1-ipso-Ar), 139.0 (G0ipso-Ar), 137.6 (p-Mes), 136.9 (ipso-Ph), 135.9 (o-Mes), 135.4 (ipso-Mes), 128.9 (m-Mes), 128.5 and $127.6\left(o-\right.$ and $m$-Ph), $127.9(p-\mathrm{Ph}), 123.5$ (Imz-C $\left.{ }^{5}\right), 120.7$ (Imz-C $\left.{ }^{4}\right), 107.3$ (G0-o-Ar), 106.4 (G1-o-Ar), 102.2 (G0-p-Ar), 101.5 (G1-p-Ar), $70.0\left(\mathrm{PhCH}_{2} \mathrm{O}\right), 69.9\left(\mathrm{G} 1-\mathrm{CH}_{2} \mathrm{O}\right), 55.0\left(\mathrm{CH}_{2}\right), 21.2$ (Mes-p-Me), 19.4 ppm (Mes-o-Me). trans-anti 11a: ${ }^{1} \mathrm{H}$ NMR $\left(\mathrm{CDCl}_{3}\right): \delta=7.40-7.25$ (m, 40H, $\left.\mathrm{Ph}\right)$, $6.78\left(\mathrm{~d},{ }^{3} J_{\mathrm{H}, \mathrm{H}}=1.8 \mathrm{~Hz}, 2 \mathrm{H}, \mathrm{Imz}-\mathrm{H}^{5}\right), 6.71-6.61\left(\mathrm{~m}, 20 \mathrm{H}, \mathrm{Imz}_{-} \mathrm{H}^{4}, \mathrm{G} 0-\right.$ and G1-o-Ar, and $\left.m-\mathrm{Mes}\right)$, $6.57\left(\mathrm{t},{ }^{4} J_{\mathrm{H}, \mathrm{H}}=2.3 \mathrm{~Hz}, 2 \mathrm{H}, \mathrm{G} 0-p-\mathrm{Ar}\right), 6.45\left(\mathrm{t},{ }^{4} J_{\mathrm{H}, \mathrm{H}}=2.3 \mathrm{~Hz}, 4 \mathrm{H}, \mathrm{G} 1-p-\mathrm{Ar}\right), 5.48\left(\mathrm{~s}, 4 \mathrm{H}, \mathrm{CH}_{2}\right), 5.00$ 
(s, 16H, PhCH $\mathrm{H}_{2} \mathrm{O}$ ), 4.92 (s, 8H, G1- $\mathrm{CH}_{2} \mathrm{O}$ ), 2.19 (s, 12H, Mes-o-Me), 2.05 ppm (s, 6H, Mes-p-Me). ${ }^{13} \mathrm{C}\left\{{ }^{1} \mathrm{H}\right\}$ NMR $\left(\mathrm{CDCl}_{3}\right): \delta=169.9$ (Imz-C $\left.{ }^{2}\right), 160.0$ (G1-m-Ar), 159.9 (G0-m-Ar), 139.6 (G1-ipsoAr), 139.1 (G0-ipso-Ar), 138.5 (p-Mes), 136.8 (ipso-Ph), 135.9 (o-Mes), 135.6 (ipso-Mes), 128.7 ( $m$-Mes), 128.6 and $127.5(o-$ and $m-\mathrm{Ph}), 128.0(p-\mathrm{Ph}), 123.9\left(\mathrm{Imz}^{-} \mathrm{C}^{5}\right), 120.4\left(\mathrm{Imz}^{-}{ }^{4}\right), 107.2(\mathrm{G} 0-$ $o$-Ar), 106.2 (G1-o-Ar), 102.1 (G0-p-Ar), 101.4 (G1-p-Ar), 70.2 (G1- $\left.\mathrm{CH}_{2} \mathrm{O}\right), 70.1$ ( $\left.\mathrm{PhCH}_{2} \mathrm{O}\right), 54.9$ $\left(\mathrm{CH}_{2}\right), 20.8$ (Me-p-Mes), 19.8 ppm (Me-o-Mes). IR (KBr pellet): $v=1595$ and 1451 (vs, C-C $\mathrm{C}_{\text {arom}}$ ), 1500 (m, CNC), 1295 (s, C-O-C $\left.\mathrm{Cas}_{\text {as }}\right), 1155$ and $1054 \mathrm{~cm}^{-1}$ (vs, C-O-C ). Elemental analysis (\%) calcd for $\mathrm{C}_{122} \mathrm{H}_{112} \mathrm{Br}_{2} \mathrm{~N}_{4} \mathrm{O}_{12} \mathrm{Pd}$ (2092.44): C 70.03, H 5.40, N 2.68; found: C 70.17, H 5.55, N 2.81. MS (ESI ${ }^{+}$-TOF in $\mathrm{CH}_{2} \mathrm{Cl}_{2} / \mathrm{MeOH} / \mathrm{NH}_{4} \mathrm{HCOO} 5 \mathrm{mM}$ ): $m / z=2106.60\left[\mathrm{M}+\mathrm{NH}_{4}\right]^{+}$.

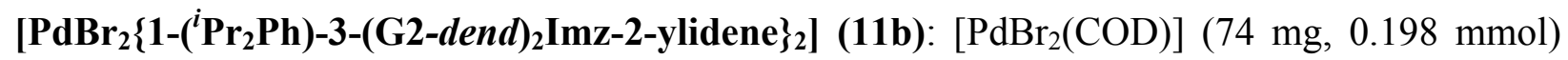
and $7 \mathbf{b}$ (453 mg, $0.396 \mathrm{mmol}$ ). Yield after purification by column chromatography: $400 \mathrm{mg}, 93 \%$. trans-syn-15: ${ }^{1} \mathrm{H}$ NMR $\left(\mathrm{CDCl}_{3}\right): \delta=7.24-7.40(\mathrm{~m}, 40 \mathrm{H}, \mathrm{Ph}), 7.07\left(\right.$ broad s, $\left.4 \mathrm{H}, m-{ }^{i} \mathrm{Pr}_{2} \mathrm{Ph}\right), 7.03$ (broad s, 2H, $p-{ }^{i} \mathrm{Pr}_{2} \mathrm{Ph}$ ), 6.79 (broad s, 2H, Imz-H ${ }^{5}$ ), 6.74 (broad s, 2H, Imz-H ${ }^{4}$ ), 6.71 (broad d, 4H, G0-o-Ar), 6.62 (d, ${ }^{3} J_{H, H}=2.2,8 \mathrm{H}, \mathrm{G} 1-o-\mathrm{Ar}$ ), 6.50 (t, ${ }^{3} J_{H, H}=2.2,4 \mathrm{H}, \mathrm{G} 1-p$-Ar), 6.44 (broad t, $2 \mathrm{H}$, G0-p-Ar), 5.76 (s, 4H, $\left.\mathrm{CH}_{2}\right), 4.97$ (s, 16H, $\left.\mathrm{PhCH}_{2} \mathrm{O}\right), 4.80$ (s, 8H, G1-CH $\left.\mathrm{C}_{2} \mathrm{O}\right), 2.78$ (sep, ${ }^{3} J_{H, H}=7.0$, $4 \mathrm{H}, \mathrm{CHMe} 2), 0.91\left(\mathrm{~d},{ }^{3} J_{H, H}=7.0,12 \mathrm{H}, \mathrm{CHMe} 2\right), 0.86\left(\mathrm{~d},{ }^{3} J_{H, H}=7.0,12 \mathrm{H}, \mathrm{CHMe} 2\right) .{ }^{13} \mathrm{C}\left\{{ }^{1} \mathrm{H}\right\} \mathrm{NMR}$ $\left(\mathrm{CDCl}_{3}\right): \delta=170.7$ (Imz-C $\left.{ }^{2}\right), 160.0$ (G0-m-Ar and G1-m-Ar), $146.5\left(p-{ }^{i} \mathrm{Pr}{ }_{2} \mathrm{Ph}\right), 139.3$ (G1-ipso-Ar), 138.9 (G0-ipso-Ar), 136.9 (ipso-Ph), 134.9 (ipso- $\left.{ }^{i} \mathrm{Pr}_{2} \mathrm{Ph}\right), 129.4\left({ }^{o}-{ }^{i} \mathrm{Pr}_{2} \mathrm{Ph}\right), 128.6$ (o-Ph), 128.0 (p$\mathrm{Ph}), 127.5(\mathrm{~m}-\mathrm{Ph}), 125.2\left(\mathrm{Imz}^{-} \mathrm{C}^{5}\right), 123.7\left(\mathrm{~m}^{i} \mathrm{Pr}_{2} \mathrm{Ph}\right), 119.0\left(\mathrm{Imz}^{-} \mathrm{C}^{4}\right), 107.0$ (G0-o-Ar), 106.4 (G1$o$-Ar), 102.3 (G0-p-Ar), 101.6 (G1-p-Ar), $70.1\left(\mathrm{Ph}-\mathrm{CH}_{2} \mathrm{O}\right), 69.9\left(\mathrm{Ar}-\mathrm{CH}_{2} \mathrm{O}\right), 55.0\left(\mathrm{CH}_{2}\right), 28.2$ $\left(\mathrm{CHMe}_{2}\right), 26.4(\mathrm{CHMe}), 23.1\left(\mathrm{CHMe}_{2}\right)$. trans-anti-15: ${ }^{1} \mathrm{H}$ NMR $\left(\mathrm{CDCl}_{3}\right): \delta=7.24-7.40(\mathrm{~m}, 40 \mathrm{H}$, $\mathrm{Ph}), 7.05$ (broad s, $4 \mathrm{H}, m-{ }^{i} \mathrm{Pr}_{2} \mathrm{Ph}$ ), 7.04 (broad s, $\left.2 \mathrm{H}, p-{ }^{i} \mathrm{Pr}_{2} \mathrm{Ph}\right), 6.67$ (broad s, 2H, Imz-H ${ }^{5}$ ), 6.74 (broad d, 4H, G0-o-Ar), $6.71\left(\mathrm{~d},{ }^{3} J_{H, H}=1.8,8 \mathrm{H}, \mathrm{G} 1-o-\mathrm{Ar}\right), 6.57\left(\operatorname{broad} \mathrm{s}, 2 \mathrm{H}, \mathrm{Imz}_{-} \mathrm{H}^{4}\right), 6.54(\mathrm{t}$, $\left.{ }^{3} J_{H, H}=1.8,4 \mathrm{H}, \mathrm{G} 1-p-\mathrm{Ar}\right), 6.50$ (broad t, $\left.2 \mathrm{H}, \mathrm{G} 0-p-\mathrm{Ar}\right), 5.41\left(\mathrm{~s}, 4 \mathrm{H}, \mathrm{CH}_{2}\right), 5.00\left(\mathrm{~s}, 16 \mathrm{H}, \mathrm{PhCH}_{2} \mathrm{O}\right)$, $4.82\left(\mathrm{~s}, 8 \mathrm{H}, \mathrm{G} 1-\mathrm{CH}_{2} \mathrm{O}\right), 3.02\left(\mathrm{sep},{ }^{3} J_{H, H}=7.0,4 \mathrm{H}, \mathrm{CHMe} 2\right), 1.11\left(\mathrm{~d},{ }^{3} J_{H, H}=7.0,12 \mathrm{H}, \mathrm{CHMe} e_{2}, 0.89\right.$ $\left(\mathrm{d},{ }^{3} J_{H, H}=7.0,12 \mathrm{H}, \mathrm{CHMe} 2\right) .{ }^{13} \mathrm{C}\left\{{ }^{1} \mathrm{H}\right\} \mathrm{NMR}\left(\mathrm{CDCl}_{3}\right): \delta=170.6\left(\mathrm{Imz}_{-} \mathrm{C}^{2}\right), 160.1(\mathrm{G} 0-m-\mathrm{Ar}$ and G1m-Ar), 146.7 ( $p$ - ${ }^{i}{ }_{2} \mathrm{Ph}$ ), 139.3 (G1-ipso-Ar), 138.9 (G0-ipso-Ar), 136.8 (ipso-Ph), 134.9 (ipso$\left.{ }^{i} \mathrm{Pr}_{2} \mathrm{Ph}\right), 129.4\left(o^{-}{ }^{i} \mathrm{Pr}_{2} \mathrm{Ph}\right), 128.6(o-\mathrm{Ph}), 128.0(p-\mathrm{Ph}), 127.5(\mathrm{~m}-\mathrm{Ph}), 125.1\left(\mathrm{Imz}-\mathrm{C}^{5}\right), 123.7(\mathrm{~m}-$ ${ }^{i} \mathrm{Pr}_{2} \mathrm{Ph}$ ), 119.0 (Imz-C ${ }^{4}$ ), 107.1 (G0-o-Ar), 106.5 (G1-o-Ar), 102.2 (G0-p-Ar), 101.5 (G1-p-Ar), 70.1 $\left(\mathrm{Ph}-\mathrm{CH}_{2} \mathrm{O}\right), 69.9\left(\mathrm{Ar}-\mathrm{CH}_{2} \mathrm{O}\right), 55.0\left(\mathrm{CH}_{2}\right), 28.6\left(\mathrm{CHMe}_{2}\right), 26.6\left(\mathrm{CHMe}_{2}\right), 22.7(\mathrm{CHMe}) . \mathrm{IR}(\mathrm{KBr}$ pellet): $v=1595(\mathrm{~m})$ and $1451\left(\mathrm{~s}, \mathrm{C}-\mathrm{C}_{\text {arom }}\right), 1497(\mathrm{~s}, \mathrm{CNC}), 1293\left(\mathrm{~m}, \mathrm{C}-\mathrm{O}-\mathrm{C}_{\mathrm{as}}\right), 1151$ and $1051 \mathrm{~cm}^{-1}$ (s, C-O-C $\mathrm{C}_{\mathrm{s}}$. Elemental analysis (\%) calcd for $\mathrm{C}_{128} \mathrm{H}_{124} \mathrm{Br}_{2} \mathrm{~N}_{4} \mathrm{O}_{12} \mathrm{Pd}(2176.60)$ : C 70.63, H 5.74, N 2.57; found: C 70.54, $\mathrm{H}$ 5.74, N 2.74. MS (ESI -TOF in $\left.\mathrm{CH}_{2} \mathrm{Cl}_{2} / \mathrm{MeOH} / \mathrm{NH}_{4} \mathrm{HCOO} 5 \mathrm{mM}\right): m / z=$ $2015.82[\mathrm{M}-2 \mathrm{Br}+\mathrm{H}]^{+}, 955.47[\mathbf{3 b}-\mathrm{Br}]^{+}$. 
[PdBr 2 \{1-Mes-3-(G3-dend)Imz-2-ylidene $\left.\}_{2}\right]$ (12a): [PdBr $\left.2(\mathrm{COD})\right]$ (19 mg, $\left.0.051 \mathrm{mmol}\right)$ and 8a (202 mg, 0.103 mmol). Yield: 173 mg, 90\%. trans-syn 12a: ${ }^{1} \mathrm{H}$ NMR $\left(\mathrm{CDCl}_{3}\right): \delta=7.40-7.25(\mathrm{~m}$, 80H, Ph), 6.79 (s, 4H, m-Mes), 6.71-6.40 (m, 46H, Imz, and G0-, G1- and G2-o and p-Ar), 5.55 (s, $\left.4 \mathrm{H}, \mathrm{CH}_{2}\right), 4.88\left(\mathrm{~s}, 32 \mathrm{H}, \mathrm{PhCH}_{2} \mathrm{O}\right), 4.80\left(\mathrm{~s}, 16 \mathrm{H}, \mathrm{G} 2-\mathrm{CH}_{2} \mathrm{O}\right), 4.71\left(\mathrm{~s}, 8 \mathrm{H}, \mathrm{G} 1-\mathrm{CH}_{2} \mathrm{O}\right), 2.40(\mathrm{~s}, 6 \mathrm{H}$, Mes-p-Me), 1.94 ppm (s, 12H, Mes-o-Me). ${ }^{13} \mathrm{C}\left\{{ }^{1} \mathrm{H}\right\}$ NMR $\left(\mathrm{CDCl}_{3}\right): \delta=170.1\left(\mathrm{Imz}^{-\mathrm{C}^{2}}\right), 160.2(\mathrm{G} 0-$ m-Ar), 160.1 (G2-m-Ar), 160.0 (G1-m-Ar), 139.4 (G1-ipso-Ar), 139.3 (G2-ipso-Ar), 139.1 (G0ipso-Ar), 137.5 (p-Mes), 136.8 (ipso-Ph), 135.9 (o-Mes), 135.4 (ipso-Mes), 128.9 (m-Mes), 128.5 and $127.5\left(o-\right.$ and $m$-Ph), $127.9(p-\mathrm{Ph}), 123.5\left(\mathrm{Imz}^{-} \mathrm{C}^{5}\right), 120.8\left(\mathrm{Imz}^{4}{ }^{4}\right), 107.0$ (G0-o-Ar), 106.4 (G2-o-Ar), 106.3 (G1-o-Ar), 102.1 (G0-p-Ar), 101.6 (G2-p-Ar), 101.4 (G1-p-Ar), 70.2 (G2-CH2 $\mathrm{O}$ ), $70.0\left(\mathrm{PhCH}_{2} \mathrm{O}\right), 69.9\left(\mathrm{G} 1-\mathrm{CH}_{2} \mathrm{O}\right), 54.8\left(\mathrm{CH}_{2}\right), 21.2$ (Mes-p-Me), 19.4 ppm (Mes-o-Me). trans-anti 12a: ${ }^{1} \mathrm{H}$ NMR $\left(\mathrm{CDCl}_{3}\right): \delta=7.40-7.25(\mathrm{~m}, 80 \mathrm{H}, \mathrm{Ph}), 6.78\left(\mathrm{~d},{ }^{3} J_{\mathrm{H}, \mathrm{H}}=1.8 \mathrm{~Hz}, 2 \mathrm{H}, \mathrm{Imz}_{-} \mathrm{H}^{5}\right), 6.71-6.61$ (m, 48H, Imz-H" ${ }^{4}, \mathrm{G} 0-, \mathrm{G} 1-$ and G2-o and $p$-Ar, and $m$-Mes), 5.45 (s, 4H, CH ), 4.99 (s, 16H, G2$\mathrm{CH}_{2} \mathrm{O}$ ), 4.95 (s, 32H, $\mathrm{PhCH} \mathrm{H}_{2} \mathrm{O}$ ), 4.88 (overlaping, 4H, G1- $\mathrm{CH}_{2} \mathrm{O}$ ), 2.15 (s, 12H, Mes-o-Me), 1.98 ppm (s, 6H, Mes- $p-M e) .{ }^{13} \mathrm{C}\left\{{ }^{1} \mathrm{H}\right\}$ NMR $\left(\mathrm{CDCl}_{3}\right): \delta=169.7\left(\mathrm{Imz}^{-} \mathrm{C}^{2}\right), 160.1$ (G2-m-Ar), 160.0 (G0m-Ar), 159.9 (G1-m-Ar), 139.6 (G1-ipso-Ar), 139.3 (G2-ipso-Ar), 139.2 (G0-ipso-Ar), 138.5 (pMes), 136.8 (ipso-Ph), 135.9 (o-Mes), 135.5 (ipso-Mes), 128.7 (m-Mes), 128.6 and 127.5 (o- and $m$-Ph), 128.0 (p-Ph), 123.9 (Imz-C ${ }^{5}$ ), 120.4 (Imz-C $\left.{ }^{4}\right), 107.0$ (G0-o-Ar), 106.4 (G2-o-Ar), 106.1 (G1-o-Ar), 102.0 (G0-p-Ar), 101.6 (G2-p-Ar), 101.3 (G1-p-Ar), 70.1 ( $\left.\mathrm{PhCH}_{2} \mathrm{O}\right), 69.8$ (G1 and G2$\mathrm{CH}_{2} \mathrm{O}$ ), $54.8\left(\mathrm{CH}_{2}\right), 20.8$ (Mes-p-Me), 19.8 ppm (Mes-o-Me). IR (KBr pellet): $v=1595$ and 1451 (vs, C-C arom), 1497 (m, CNC), $1295\left(\mathrm{~m}, \mathrm{C}-\mathrm{O}-\mathrm{C}_{\mathrm{as}}\right), 1155$ and $1051 \mathrm{~cm}^{-1}$ (vs, C-O-C $)_{\mathrm{s}}$. Elemental analysis (\%) calcd for $\mathrm{C}_{234} \mathrm{H}_{208} \mathrm{Br}_{2} \mathrm{~N}_{4} \mathrm{O}_{28} \mathrm{Pd}$ (3790.39): C 74.15, H 5.53, N 1.48; found: $\mathrm{C} 73.20, \mathrm{H}$ 5.68, $\mathrm{N}$ 1.47. MS (ESI ${ }^{+}$-TOF in $\mathrm{CH}_{2} \mathrm{Cl}_{2} / \mathrm{MeOH} / \mathrm{NH}_{4} \mathrm{HCOO} 5 \mathrm{mM}$ ) was not informative.

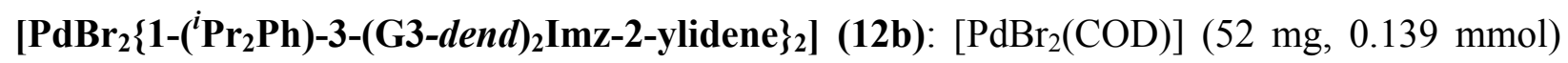
and $8 \mathbf{b}$ (550 mg, $0.276 \mathrm{mmol}$ ). Yield after purification by column chromatography: $390 \mathrm{mg}, 73 \%$. trans-syn-12b: ${ }^{1} \mathrm{H}$ NMR $\left(\mathrm{CDCl}_{3}\right): \delta=7.24-7.40(\mathrm{~m}, 80 \mathrm{H}, \mathrm{Ph}), 7.04$ (broad s, $\left.4 \mathrm{H}, m-{ }^{i} \mathrm{Pr}_{2} \mathrm{Ph}\right), 7.02$ (broad s, 2H, $p-{ }^{i} \mathrm{Pr}_{2} \mathrm{Ph}$ ), 6.72 (broad s, 2H, Imz-H ${ }^{5}$ ), 6.36-7.31 (m, 44H, G0-o-Ar, G1-o-Ar, G2-oAr, G0-p-Ar, G1-p-Ar, G2-p-Ar, and Imz-H ${ }^{4}$ ), 5.73 (s, 4H, $\mathrm{CH}_{2}$ ), 4.97 (s, 8H, G2-CH $\mathrm{H}_{2} \mathrm{O}$ ), 4.89 (s, $\left.16 \mathrm{H}, \mathrm{PhCH}_{2} \mathrm{O}\right), 4.81\left(\mathrm{~s}, 8 \mathrm{H}, \mathrm{G} 1-\mathrm{CH}_{2} \mathrm{O}\right), 2.80(\mathrm{~m}, 4 \mathrm{H}, \mathrm{CHMe}), 0.89\left(\mathrm{~d},{ }^{3} J_{H, H}=6.9,12 \mathrm{H}, \mathrm{CHMe} e_{2}\right)$, $0.83\left(\mathrm{~d},{ }^{3} J_{H, H}=6.9,12 \mathrm{H}, \mathrm{CHMe} 2\right) .{ }^{13} \mathrm{C}\left\{{ }^{1} \mathrm{H}\right\} \mathrm{NMR}\left(\mathrm{CDCl}_{3}\right): \delta=170.0\left(\mathrm{Imz}^{-\mathrm{C}}{ }^{2}\right), 160.0(\mathrm{G} 2-m-\mathrm{Ar})$, 159.9 (G0-m-Ar and G1-m-Ar overlapping), 140.0 ( $\left.p-{ }^{i} \mathrm{Pr}_{2} \mathrm{Ph}\right), 139.3$ (G1-ipso-Ar), 139.2 (G2-ipsoAr), 138.9 (G0-ipso-Ar), 136.7 (ipso- $\mathrm{C}_{6} \mathrm{H}_{3}{ }^{i} \mathrm{Pr}_{2}$ and ipso-Ph overlapping), 128.5 (o-Ph and $o$ - ${ }^{i} \mathrm{Pr}_{2} \mathrm{Ph}$ overlapping), $127.4(p-\mathrm{Ph}), 127.5(m-\mathrm{Ph}), 125.1\left(m-{ }^{i} \mathrm{Pr}_{2} \mathrm{Ph}\right.$ and Imz-C ${ }^{5}$ overlapping), 120.2 (Imz$\mathrm{C}^{4}$ ), 106.3 (G0-o-Ar, G1-o-Ar and G2-o-Ar overlapping), 101.5 (G0-p-Ar, G1-p-Ar and G2-p-Ar overlapping), $69.9\left(\mathrm{Ph}-\mathrm{CH}_{2} \mathrm{O}\right), 69.7$ (G1-Ar- $\mathrm{CH}_{2} \mathrm{O}$ and $\mathrm{G} 2-\mathrm{Ar}-\mathrm{CH}_{2} \mathrm{O}$ overlapping), $54.4\left(\mathrm{CH}_{2}\right)$, $\left.29.7\left(\mathrm{CHMe}_{2}\right), 26.4(\mathrm{CHMe}), 23.2(\mathrm{CHMe})_{2}\right)$ trans-anti-16: ${ }^{1} \mathrm{H} \mathrm{NMR}\left(\mathrm{CDCl}_{3}\right): \delta=7.24-7.40(\mathrm{~m}$, 
80H, Ph), 7.02 (broad s, 4H, $m-{ }^{i}{ }^{P}{ }_{2} \mathrm{Ph}$ ), 7.01 (broad s, 2H, $p-{ }^{i} \mathrm{Pr}_{2} \mathrm{Ph}$ ), 6.36-7.31 (m, 46H, G0-o-Ar, G1-o-Ar, G2-o-Ar, G0-p-Ar, G1-p-Ar, G2-p-Ar, Imz-H ${ }^{4}$, and Imz-H ${ }^{5}$ ), 5.42 (s, 4H, $\mathrm{CH}_{2}$ ), 4.99 (s, $\left.8 \mathrm{H}, \mathrm{G} 2-\mathrm{CH}_{2} \mathrm{O}\right), 4.91$ (s, 16H, $\left.\mathrm{PhCH}_{2} \mathrm{O}\right), 4.74\left(\mathrm{~s}, 8 \mathrm{H}, \mathrm{G} 1-\mathrm{CH}_{2} \mathrm{O}\right), 3.02(\mathrm{~m}, 4 \mathrm{H}, \mathrm{CHMe}$ ), 1.20 (d, $\left.{ }^{3} J_{H, H}=6.9,12 \mathrm{H}, \mathrm{CHMe} 2\right), 0.81\left(\mathrm{~d},{ }^{3} J_{H, H}=6.9,12 \mathrm{H}, \mathrm{CHMe} 2\right) .{ }^{13} \mathrm{C}\left\{{ }^{1} \mathrm{H}\right\} \mathrm{NMR}\left(\mathrm{CDCl}_{3}\right): \delta=170.0$ (Imz-C ${ }^{2}$ ), 160.0 (G2-m-Ar), 159.8 (G0-m-Ar and G1-m-Ar overlapping), $140.0\left(p-{ }^{i} \operatorname{Pr}_{2} \mathrm{Ph}\right), 139.3$ (G1-ipso-Ar), 139.2 (G2-ipso-Ar), 138.9 (G0-ipso-Ar), $136.7 \quad$ (ipso- ${ }^{i} \mathrm{Pr}_{2} \mathrm{Ph}$ and ipso-Ph overlapping), 128.5 (o-Ph and $o-{ }^{i}{ }^{P} r_{2} \mathrm{Ph}$ overlapping), $127.5(m-\mathrm{Ph}), 127.4(p-\mathrm{Ph}), 125.1\left(m-{ }^{i} \operatorname{Pr}_{2} \mathrm{Ph}\right.$ and Imz-C $\mathrm{C}^{5}$ overlapping), $120.2\left(\mathrm{Imz}^{4} \mathrm{C}^{4}\right), 106.9$ (G0-o-Ar, G1-o-Ar and G2-o-Ar overlapping), 101.6 (G0-p-Ar, G1-p-Ar and G2-p-Ar overlapping), $69.9\left(\mathrm{Ph}-\mathrm{CH}_{2} \mathrm{O}\right), 69.7$ (G1-Ar- $\mathrm{CH}_{2} \mathrm{O}$ and G2Ar- $\mathrm{CH}_{2} \mathrm{O}$ overlapping), $54.4\left(\mathrm{CH}_{2}\right), 29.6\left(\mathrm{CHMe}_{2}\right), 26.6(\mathrm{CHMe}), 23.1(\mathrm{CHMe})$. IR ( $\mathrm{KBr}$ pellet): $v=1595(\mathrm{~m})$ and $1451\left(\mathrm{~s}, \mathrm{C}-\mathrm{C}_{\mathrm{arom}}\right), 1496(\mathrm{~s}, \mathrm{CNC}), 1291\left(\mathrm{~m}, \mathrm{C}-\mathrm{O}-\mathrm{C}_{\mathrm{as}}\right), 1151$ and $1054 \mathrm{~cm}^{-1}(\mathrm{~s}, \mathrm{C}-$ $\mathrm{O}-\mathrm{C}_{\mathrm{s}}$ ). Elemental analysis (\%) calcd for $\mathrm{C}_{240} \mathrm{H}_{220} \mathrm{Br}_{2} \mathrm{~N}_{4} \mathrm{O}_{28} \mathrm{Pd}$ (3874.55): C 74.40, H 5.72, N 1.45; found: $\mathrm{C}$ 74.51, $\mathrm{H}$ 5.79, $\mathrm{N}$ 1.57. $\mathrm{MS}\left(\mathrm{ESI}^{+}-\mathrm{TOF}\right.$ in $\left.\mathrm{CH}_{2} \mathrm{Cl}_{2} / \mathrm{MeOH} / \mathrm{NH}_{4} \mathrm{HCOO} 5 \mathrm{mM}\right): \mathrm{m} / z=$ $3729.53\left[\mathrm{M}-2 \mathrm{Br}+\mathrm{NH}_{4}\right]^{+}$. 


\section{X-ray crystallographic studies.}

Single crystals suitable for X-ray diffraction studies were obtained by slow evaporation from acetone (5a) or from $\mathrm{CH}_{2} \mathrm{Cl}_{2} /$ hexane (5b) solutions, or by slow diffusion of hexane into a dichloromethane solution of the complex (6b, trans-anti-9a and trans-syn-10a) at room temperature. Crystals of diastereomer cis-anti-9a were obtained by recrystallization of $9 \mathbf{a}$ in $\mathrm{CH}_{2} \mathrm{Cl}_{2}$ after standing the solution in a freezer at $-20{ }^{\circ} \mathrm{C}$ for 2 weeks. A summary of crystal data, data collection, and refinement parameters for the structural analysis is given in Table S1 (for $\mathbf{5 a}, \mathbf{5 b}$ and 6b) and Table S2 (trans-anti-9a, cis-anti-9a, and trans-syn 10a). Unfortunately, good refinement of cis-9a was not possible due to deficient quality of the crystals and collected data (a representation of the molecular structure of this complex is shown in Figure S1). Suitable crystals were covered with mineral oil and mounted in the $\mathrm{N}_{2}$ stream of a Bruker-Nonius Kappa-CCD diffractometer with area detector, and equipped with an Oxford Cryostream 700 unit; data were collected using graphite monochromated Mo- $K_{a}$ radiation $(\lambda=0.71073 \AA)$ at $200 \mathrm{~K}$, with an exposure time of $10 \mathrm{~s}$ per frame (four sets; 295 frames; phi and omega scan, 2.0 scan-width) for compound 5a; an exposure time of 169 s per frame (four sets; 351 frames; phi and omega scans $1.70^{\circ}$ scan-width) for compound $\mathbf{5 b}$; an exposure time of $89 \mathrm{~s}$ per frame (five sets; 645 frames; phi and omega scans $1.0^{\circ}$ scan-width) for compound $\mathbf{6 b}$; an exposure time of $67 \mathrm{~s}$ per frame (four sets; 271 frames; phi and omega scans $2.00^{\circ}$ scan-width) for compound trans-9a; an exposure time of $21 \mathrm{~s}$ per frame (six sets; 1385 frames; phi and omega scans $0.70^{\circ}$ scan-width) for compound cis-9a; and an exposure time of $143 \mathrm{~s}$ per frame (four sets; 334 frames; phi and omega scans $1.60^{\circ}$ scan-width) for compound 10a. Raw data were corrected for Lorenz and polarization effects.

Structures were solved by direct methods, completed by the subsequent difference Fourier techniques and refined by full-matrix least squares on $F^{2}$ (SHELXL-97). ${ }^{\text {S5 }}$ Anisotropic thermal parameters were used in the last cycles of refinement for the non hydrogen atoms in both structures. The hydrogen atoms were introduced in the last cycle of refinement from geometrical calculations and refined using a riding model. All the calculations were made using the WINGX program. ${ }^{\text {S6 }}$

CCDC-1435764 (for 5a), CCDC-1435765 (for 5b), CCDC-1435766 (for 6b), CCDC-1435768 (for trans-9a), CCDC-1435767 (for cis-9a), and CCDC-1435769 (for 10a) contain the supplementary crystallographic data for this paper. These data can be obtained free of charge from The Cambridge Crystallographic Data Centre via www.ccdc.cam.ac.uk/data_request/cif. 
Table S1. Crystal data and structure refinement for silver compounds $\mathbf{5 a}, \mathbf{5 b}$ and $\mathbf{6 b}$.

\begin{tabular}{|c|c|c|c|}
\hline Compound & 5a & $5 \mathbf{b}$ & $6 \mathbf{b}$ \\
\hline Empirical formula & $\mathrm{C}_{19} \mathrm{H}_{20} \mathrm{Ag} \mathrm{BrN} \mathrm{Br}_{2}$ & $\mathrm{C}_{44} \mathrm{H}_{52} \mathrm{Ag}_{2} \mathrm{Br}_{2} \mathrm{~N}_{4}$ & $\mathrm{C}_{36} \mathrm{H}_{38} \mathrm{AgBrN}_{2} \mathrm{O}_{2}$ \\
\hline Formula weight & 464.15 & 1012.46 & 718.46 \\
\hline Crystal size $[\mathrm{mm}]$ & $0.30 \times 0.20 \times 0.15$ & $0.32 \times 0.25 \times 0.10$ & $0.40 \times 0.332 \times 0.23$ \\
\hline Color & colorless & colorless & colorless \\
\hline Temperature $[\mathrm{K}]$ & $200(2)$ & $200(2)$ & $200(2)$ \\
\hline Wavelength $[\AA]$ & 0.71073 & 0.71073 & 0.71073 \\
\hline Crystal system, space group & monoclinic, $P 2_{1} / a$ & monoclinic, $P n$ & monoclinic, $P 2_{1} / c$ \\
\hline$a[\AA]$ & $10.052(5)$ & $9.7047(4)$ & $8.607(2)$ \\
\hline$b[\AA]$ & $17.616(5)$ & $10.9618(9)$ & $29.518(6)$ \\
\hline$c[\AA]$ & $10.466(5)$ & $20.7150(16)$ & $15.321(3)$ \\
\hline$\alpha\left[^{\circ}\right]$ & 90.0 & 90.0 & 90.0 \\
\hline$\beta\left[^{\circ}\right]$ & $95.319(5)$ & $102.546(4)$ & $122.845(13)$ \\
\hline$\gamma\left[\left[^{\circ}\right]\right.$ & 90.0 & 90.0 & 90.0 \\
\hline Volume $\left[\AA^{3}\right]$ & $1845.3(14)$ & 2151.1(3) & $3270.1(12)$ \\
\hline$Z$, Calcd. density $\left[\mathrm{g} / \mathrm{cm}^{3}\right]$ & $4,1.671$ & $2,1.563$ & $4,1.459$ \\
\hline Absorption coeff. $\left[\mathrm{mm}^{-1}\right]$ & 3.258 & 2.802 & 1.872 \\
\hline$F(000)$ & 920 & 1016 & 1464 \\
\hline$\theta$ range $\left[{ }^{\circ}\right]$ & 3.03 to 27.50 & 3.17 to 27.51 & 3.14 to 27.59 \\
\hline Limiting indices & $\begin{array}{l}-13 \leq h \leq 13 \\
-22 \leq k \leq 22 \\
-13 \leq l \leq 13\end{array}$ & $\begin{array}{l}-12 \leq h \leq 12 \\
-14 \leq k \leq 14 \\
-26 \leq l \leq 26\end{array}$ & $\begin{array}{l}-11 \leq h \leq 11 \\
-38 \leq k \leq 38 \\
-19 \leq l \leq 18\end{array}$ \\
\hline Reflections collected/unique & $\begin{array}{l}15474 / 4239 \\
{[R(\text { int })=0.0842]}\end{array}$ & $\begin{array}{l}17827 / 9825 \\
{[\mathrm{R}(\text { int })=0.0525]}\end{array}$ & $\begin{array}{l}45689 / 7518 \\
{[R(\text { int })=0.0835}\end{array}$ \\
\hline Completeness to $\theta$ & $27.52, \quad 99.7 \%$ & $27.51, \quad 99.8 \%$ & $27.59, \quad 99.1 \%$ \\
\hline Absorption correction & $\begin{array}{l}\text { Semi-empirical from } \\
\text { equivalents }\end{array}$ & $\begin{array}{l}\text { Semi-empirical from } \\
\text { equivalents }\end{array}$ & $\begin{array}{l}\text { Semi-empirical from } \\
\text { equivalents }\end{array}$ \\
\hline Max. and min. transmission & 0.553 and 0.435 & 0.343 and 0.278 & 0.778 and 0.559 \\
\hline Refinement method & $\begin{array}{l}\text { Full-matrix least- } \\
\text { squares on } F^{2}\end{array}$ & $\begin{array}{l}\text { Full-matrix least- } \\
\text { squares on } F^{2}\end{array}$ & $\begin{array}{l}\text { Full-matrix least- } \\
\text { squares on } F^{2}\end{array}$ \\
\hline Data/restraints/parameters & $4239 / 0 / 208$ & $9825 / 2 / 469$ & $7518 / 0 / 379$ \\
\hline Goodness of fit on $F^{2}$ & 0.987 & 0.976 & 1.082 \\
\hline$R 1 / w R 2[I>2 \mathrm{~s}(I)]$ & $0.0435 / 0.0878$ & $0.0389 / 0.0793$ & $0.0597 / 0.1221$ \\
\hline$R 1 / w R 2$ (all data) & $0.0907 / 0.0991$ & $0.0681 / 0.0869$ & $0.1105 / 0.1471$ \\
\hline $\begin{array}{l}\text { Largest diff. peak and hole } \\
{\left[\mathrm{e} / \AA^{3}\right]}\end{array}$ & 0.661 and -1.138 & 0.0625 and -0.0877 & 2.478 and -0.713 \\
\hline
\end{tabular}


Table S2. Crystal data and structure refinement for palladium compounds trans-anti-9a, cis-anti-9a, and trans-syn-10a.

\begin{tabular}{|c|c|c|c|}
\hline Compound & trans-anti-9a & cis-sym-9a & $10 a$ \\
\hline Empirical formula & $\mathrm{C}_{38} \mathrm{H}_{40} \mathrm{Br}_{2} \mathrm{~N}_{4} \mathrm{Pd}$ & $\mathrm{C}_{38} \mathrm{H}_{40} \mathrm{Br}_{2} \mathrm{~N}_{4} \mathrm{Pd}$ & $\mathrm{C}_{72} \mathrm{H}_{70} \mathrm{Br}_{2} \mathrm{~N}_{4} \mathrm{O}_{4} \mathrm{Pd}$ \\
\hline Formula weight & 818.96 & 818.96 & 1321.54 \\
\hline Crystal size $[\mathrm{mm}]$ & $0.50 \times 0.40 \times 0.10$ & $0.25 \times 0.20 \times 0.15$ & $0.33 \times 0.26 \times 0.13$ \\
\hline Color & yellow & yellow & yellow \\
\hline Temperature $[\mathrm{K}]$ & $200(2)$ & $200(2)$ & $200(2)$ \\
\hline Wavelength $[\AA]$ & 0.71069 & 0.71073 & 0.71073 \\
\hline Crystal system, space group & triclinic, $P-1$ & monoclinic, $P 2_{1} / n$ & triclinic, $P-1$ \\
\hline$a[\AA]$ & $8.374(5)$ & $15.694(14)$ & $11.843(2)$ \\
\hline$b[\AA]$ & $10.292(5)$ & $18.447(15)$ & $12.7275(18)$ \\
\hline$c[\AA]$ & $11.427(5)$ & $39.27(3)$ & $21.447(4)$ \\
\hline$\alpha\left[^{\circ}\right]$ & $88.822(5)$ & 90.0 & $75.544(10)$ \\
\hline$\beta\left[^{\circ}\right]$ & $78.895(5)$ & $89.46(7)$ & $83.173(13)$ \\
\hline$\gamma\left[^{\circ}\right]$ & $68.837(5)$ & 90.0 & $81.698(12)$ \\
\hline Volume $\left[\AA^{3}\right]$ & $899.9(8)$ & $11368(16)$ & $3086.1(9)$ \\
\hline$Z$, Calcd. density $\left[\mathrm{g} / \mathrm{cm}^{3}\right]$ & $1,1.511$ & $12,1.436$ & $2,1.422$ \\
\hline Absorption coeff. $\left[\mathrm{mm}^{-1}\right]$ & 2.767 & 2.629 & 1.649 \\
\hline$F(000)$ & 412 & 4944 & 1356 \\
\hline$\theta$ ranges $\left[{ }^{\circ}\right]$ & 3.05 to 27.50 & 2.99 to 25.05 & 3.03 to 27.50 \\
\hline Limiting indices & $\begin{array}{l}-10 \leq h \leq 10 \\
-13 \leq k \leq 13 \\
-14 \leq l \leq 14\end{array}$ & $\begin{array}{l}-17 \leq h \leq 18 \\
-21 \leq k \leq 20 \\
-46 \leq l \leq 44\end{array}$ & $\begin{array}{l}-15 \leq h \leq 15 \\
-16 \leq k \leq 16 \\
-27 \leq l \leq 26\end{array}$ \\
\hline Reflections collected/unique & $\begin{array}{l}7706 / 4102 \\
{[R(\text { int })=0.0472]}\end{array}$ & $\begin{array}{l}45869 / 18803 \\
{[\mathrm{R}(\text { int })=0.3356]}\end{array}$ & $\begin{array}{l}26585 / 14135 \\
{[R(\text { int })=0.0733]}\end{array}$ \\
\hline Completeness to $\theta$ & $27.50 \quad 99.4 \%$ & $25.05 \quad 93.4 \%$ & $27.50 \quad 99.7 \%$ \\
\hline Absorption correction & $\begin{array}{l}\text { Semi-empirical from } \\
\text { equivalents }\end{array}$ & $\begin{array}{l}\text { Semi-empirical from } \\
\text { equivalents }\end{array}$ & $\begin{array}{l}\text { Semi-empirical from } \\
\text { equivalents }\end{array}$ \\
\hline Max. and min. transmission & 1.07 and 0.949 & 1.372 and 0.829 & 0.8142 and 0.6122 \\
\hline Refinement method & $\begin{array}{l}\text { Full-matrix least- } \\
\text { squares on } F^{2}\end{array}$ & $\begin{array}{l}\text { Full-matrix least- } \\
\text { squares on } F^{2}\end{array}$ & $\begin{array}{l}\text { Full-matrix least- } \\
\text { squares on } F^{2}\end{array}$ \\
\hline Data/restraints/parameters & $4102 / 0 / 205$ & $18803 / 0 / 617$ & $14135 / 0 / 748$ \\
\hline Goodness of fit on $F^{2}$ & 0.975 & 0.987 & 0.884 \\
\hline$R 1 / w R 2[I>2 \mathrm{~s}(I)]$ & $0.0371 / 0.0794$ & $0.1479 / 0.3318$ & $0.0450 / 0.0729$ \\
\hline$R 1 / w R 2$ (all data) & $0.0658 / 0.0876$ & $0.2985 / 0.4288$ & $0.1205 / 0.0871$ \\
\hline $\begin{array}{l}\text { Largest diff. peak and hole } \\
{\left[\mathrm{e} / \AA^{3}\right]}\end{array}$ & 0.694 and -1.102 & 2.62 and -1.718 & 0.595 and -0.692 \\
\hline
\end{tabular}


Figure S1. Molecular structure found for cis-anti-9a (H omitted for clarity). Selected bond lengths $[\AA]$ and angles $\left[^{\circ}\right]$ in the form of ranges due to the presence of three independent molecules in the asymmetric unit: $\quad \operatorname{Pd}(1)-\mathrm{C}(1) \quad 1.96(2)-2.00(3), \quad \operatorname{Pd}(1)-\mathrm{C}(4) \quad 1.99(2)-2.00(3), \quad \operatorname{Pd}(1)-\operatorname{Br}(1)$ 2.485(3)-2.514(3), $\operatorname{Pd}(1)-\operatorname{Br}(2) \quad 2.497(3)-2.516(4) ; \quad \operatorname{Br}(1)-\operatorname{Pd}(1)-\operatorname{Br}(2) \quad 89.8(1)-91.8(1), \quad C(1)-$ $\operatorname{Pd}(1)-\mathrm{C}(4) \quad$ 88.0(9)-92.0(1), $\quad \mathrm{C}(1)-\operatorname{Pd}(1)-\operatorname{Br}(1) \quad 87.1(8)-91.0(7), \quad \mathrm{C}(1)-\operatorname{Pd}(1)-\operatorname{Br}(2)$ 172.5(8)-176.8(7), C(4)-Pd(1)-Br(1) 175.4(6)-178.9(6), C(4)-Pd(1)-Br(2) 90.4(5)-91.6(6).

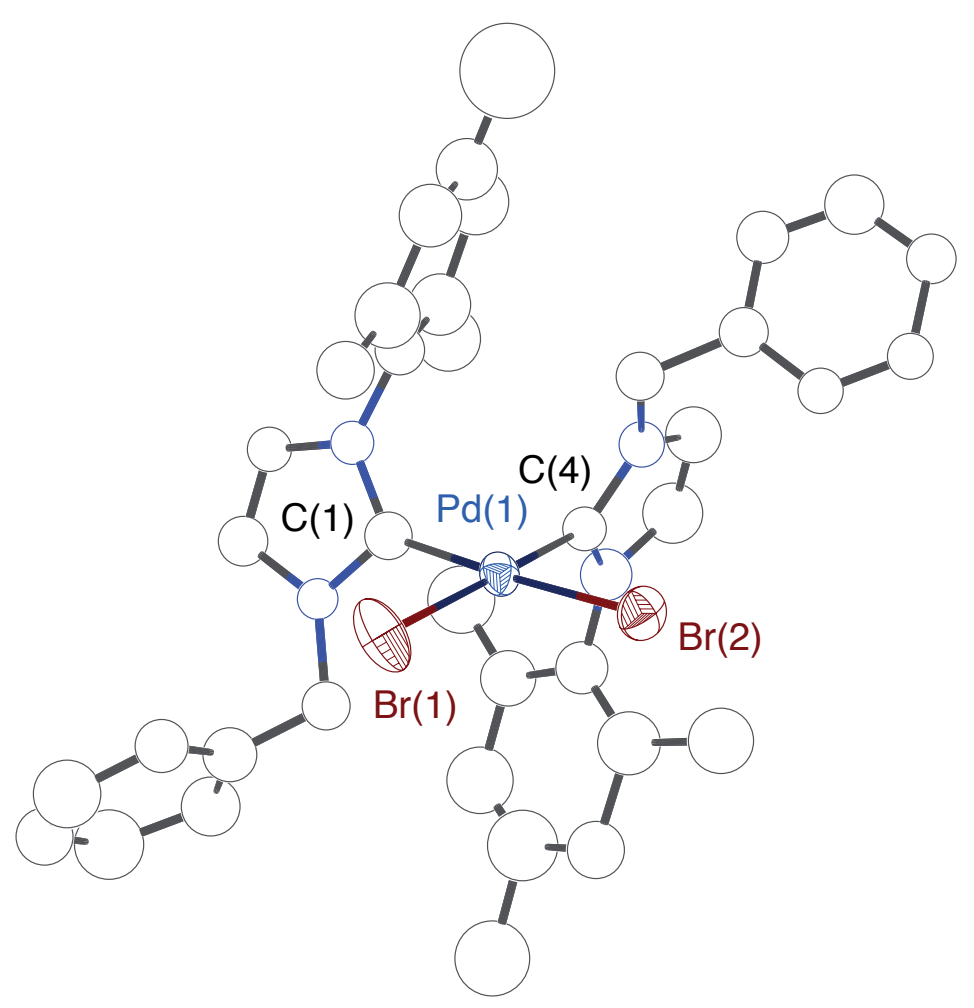




\section{General procedure for Heck reactions}

Prior to use, all glassware and materials in contact with the catalytic mixtures were treated with aqua regia to remove any possible palladium residue. Reaction blanks, without catalyst, were performed to rule out false positives due to possible contamination by palladium from the reactants. All catalytic assays were carried out under argon into $10-\mathrm{mL}$ screw-capped glass vials equipped with a magnetic stirrer, in DMF or acetonitrile solutions $(5 \mathrm{~mL})$ containing triethylamine $(0.6$ mmol, $0.12 \mathrm{M})$, methyl acrylate $(0.6 \mathrm{mmol}, 0.12 \mathrm{M})$, 4-iodotoluene $(0.5 \mathrm{mmol}, 0.10 \mathrm{M})$, naphthalene $(0.5 \mathrm{mmol}, 0.10 \mathrm{M})$, and the corresponding amount of catalyst for Pd-loadings of 1.0, 0.5, 0.1 or 0.01 mol-\% $\%$ (5.0, 2.5, 0.5 or $0.05 \mu \mathrm{mol}$, respectively). The vials were immediately placed in an oil bath thermostatted at $130{ }^{\circ} \mathrm{C}$ for the reactions in DMF, or at $80{ }^{\circ} \mathrm{C}$ for those in acetonitrile, with vigorous stirring, taking that instant as the starting time of the reaction. Stirring of the mixture was maintained, and the progress of the reaction monitored by GC with periodic withdrawal of $1 \mu \mathrm{L}$ of the catalytic mixture. Conversions (\%) were determined taking naphthalene as the internal standard and the corresponding calibration curve for 4-iodotoluene. All runs were performed at least two times with reproducibility of the conversions measured within $\pm 3 \%$. For the catalysts reutilization experiments, once the reactions in DMF were complete, the vials were reloaded at room temperature with methyl acrylate, 4-iodotoluene, and triethylamine in amounts identical to those initially added (specified above). The vials were then again placed in the oil bath at $130{ }^{\circ} \mathrm{C}$ taking that instant as the new starting time. 


\section{Fluorescence Methods}

Absorption spectra were recorded in a Perkin-Elmer, Lambda35 UV-Vis spectrophotometer. Steady-state fluorescence measurements were performed by using a high sensitivity spectrofluorometer, the SLM 8100C AMINCO, equipped with a cooled photomultiplier and a double (single) monochromator in the excitation (emission) path. Slit widths were selected at $8 \mathrm{~nm}$ for both excitation and emission channels and polarizers were placed at the magic angle conditions. The cell housing temperature was well controlled by using a thermostatic bath (Huber Ministat) and measured by employing a Pt-100 probe with digital temperature processor (Nokeval, model 2011). The fluorescence decay measurements were obtained with a Time Correlated Single Photon Counting (TCSPC) FL900 Edinburgh Instruments Spectrometer. Excitation was carried out by using nanoleds (IBH) emitting at 253 and $335 \mathrm{~nm}$. The system was equipped with two concave grating monochromators at both the excitation and emission paths and a red sensitive photomultiplier also immersed in a Peltier cooled housing. No polarizers were used in these experiments. Data acquisition was carried out by using a multichannel time detector and a time window width of $125 \mathrm{~ns}$, with a total of 10,000 counts at the intensity maximum. The instrumental response function was regularly obtained by measuring the scattering of a Ludox solution. Temperature was controlled by a bath (Techne, TE-8A).

Right angle geometry and quartz $10 \mathrm{~mm}$ path cells were used for fluorescence experiments.

The amount of intramolecular excimers for $\mathrm{G} 0-\mathrm{G} 3(\mathrm{G \#})$ was quantified by the ratio of excimers and monomer emission intensities, $\mathrm{I}_{\mathrm{E}} / \mathrm{I}_{\mathrm{M}}$, which was corrected by subtracting the fluorescence of the monomer at the excimer wavelength according to:

$$
\mathrm{I}_{\mathrm{E}} / \mathrm{I}_{\mathrm{M}}=\left(\mathrm{I}_{\mathrm{G} \#, \lambda_{\mathrm{E}}}-\mathrm{I}_{\mathrm{mon}, \lambda}\right) / \mathrm{I}_{\mathrm{M}(\text { norm })}
$$

where $I_{G \#, \lambda_{E}}$ is the normalized fluorescence intensity for $G \#$ at the wavelength of the excimer band, $\lambda_{\mathrm{E}} \cdot \mathrm{I}_{\mathrm{mon}, \lambda_{\mathrm{E}}}$ is the normalized intensity obtained for benzene model compound which here was taken as zero and $\mathrm{I}_{\mathrm{M}(\text { norm })}$ is the intensity at the monomer wavelength that is used for normalization of the emission spectra.

Decay intensity profiles were fitted to a sum of exponential decay functions as

$$
\mathrm{I}(\mathrm{t})=\sum_{\mathrm{i}=1}^{\mathrm{n}} \mathrm{A}_{\mathrm{i}} \mathrm{e}^{-\mathrm{t} / \tau_{\mathrm{i}}}
$$


by the iterative reconvolution method. ${ }^{\mathrm{S} 7}$ The average lifetime of a multiple-exponential decay function was then defined as

$$
\langle\tau\rangle=\frac{\sum_{\mathrm{i}=1}^{\mathrm{n}} \mathrm{A}_{\mathrm{i}} \tau_{\mathrm{i}}^{2}}{\sum_{\mathrm{i}=1}^{\mathrm{n}} \mathrm{A}_{\mathrm{i}} \tau_{\mathrm{i}}}
$$

where $A_{i}$ is the pre-exponential factor of the component with a lifetime $\tau_{i}$ of the multi-exponential function intensity decay.

For a single excited species which is dynamically quenched with $\mathrm{Q}$, the ratio of $\tau$ in the presence of $\mathrm{Q}$ and its absence $\left(\tau_{\mathrm{q}=0}\right)$ are related with [Q] by the well-know linear Stern-Volmer (SV) equation. The slope of linear Stern-Volmer plots provides the quenching constant $\mathrm{K}_{\mathrm{SV}}$, which is also related to a bimolecular quenching constant $\mathrm{k}_{\mathrm{q}}$ and lifetime in the absence of quencher $\tau_{\mathrm{q}=0}$. For complex systems, the Stern-Volmer representations of $\langle\tau\rangle \mid\left\langle\tau_{0}\right\rangle$ vs [Q] are only linear at low quencher concentrations. ${ }^{\text {S8 }}$ 
Figure S2. Absorption spectra for 9a-12a dendrimer series in $\mathrm{CH}_{2} \mathrm{Cl}_{2}$ at $25{ }^{\circ} \mathrm{C}$. Superimposed the 280-480 $\mathrm{nm}$ absorption region is enlarged. Spectrum for $\mathrm{PdBr}_{2}(\mathrm{cod})$ is included.

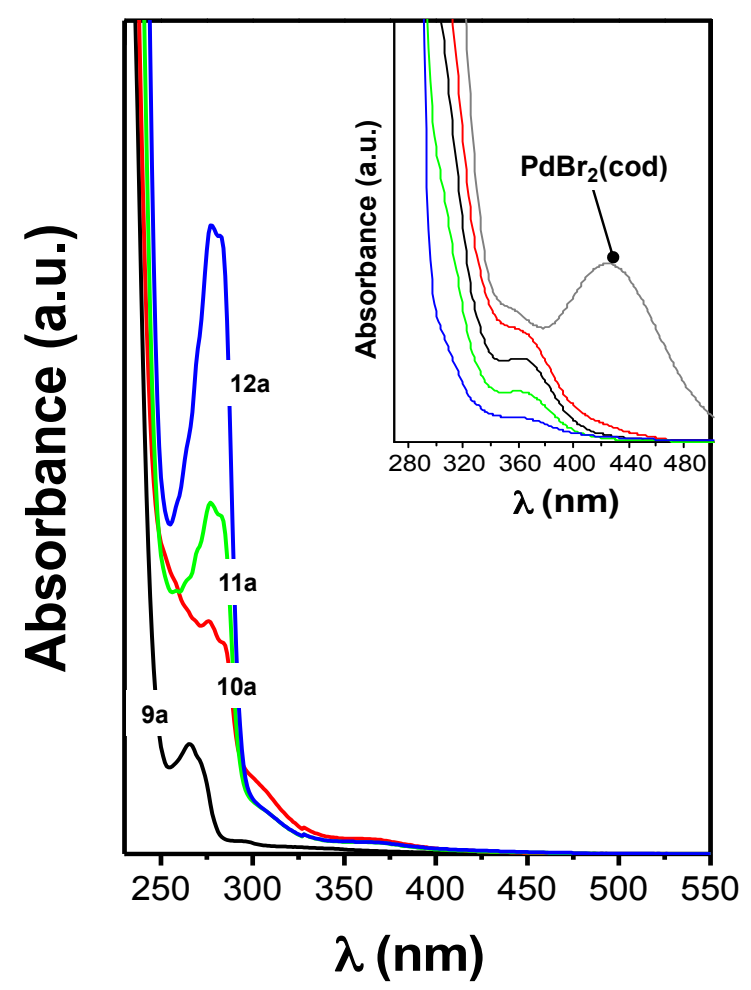

Table S3. Some photophysical parameters for $\mathrm{G} n(\mathbf{9 a}-\mathbf{1 2 a})$ dendrimers in $\mathrm{CH}_{2} \mathrm{Cl}_{2}$ at $25^{\circ} \mathrm{C}$.

\begin{tabular}{cccc}
\hline Dendrimer & $\mathbf{1 0}^{\mathbf{3}} \boldsymbol{\Phi}_{\mathbf{3 4 0 n m}}$ & $\mathbf{I}_{\mathbf{E}} / \mathbf{I}_{\mathbf{M}}$ & $<\boldsymbol{\tau}>\mathbf{( n s )}$ \\
\hline $\mathbf{9 a}$ & 0.5 & 0.28 & 4.4 \\
$\mathbf{1 0 a}$ & 3.0 & 3.02 & 3.9 \\
$\mathbf{1 1 a}$ & 1.3 & 0.17 & 3.0 \\
$\mathbf{1 2 a}$ & 1.3 & 0.13 & 3.1
\end{tabular}


Figure S3. Stern-Volmer plots of the fluorescence intensity measured at the maximum of emission band $(420 \mathrm{~nm})$ from the Pd-core for dilute solutions of 9a-12a dendrimers in $\mathrm{CH}_{2} \mathrm{Cl}_{2}$ at $25{ }^{\circ} \mathrm{C}$. The quencher was diacetyl. Symbols are: 9a $(\mathbf{\Delta})$; 10a $(\bullet)$; 11a $(\mathbf{\square})$ and 12a $(\boldsymbol{\nabla})$.

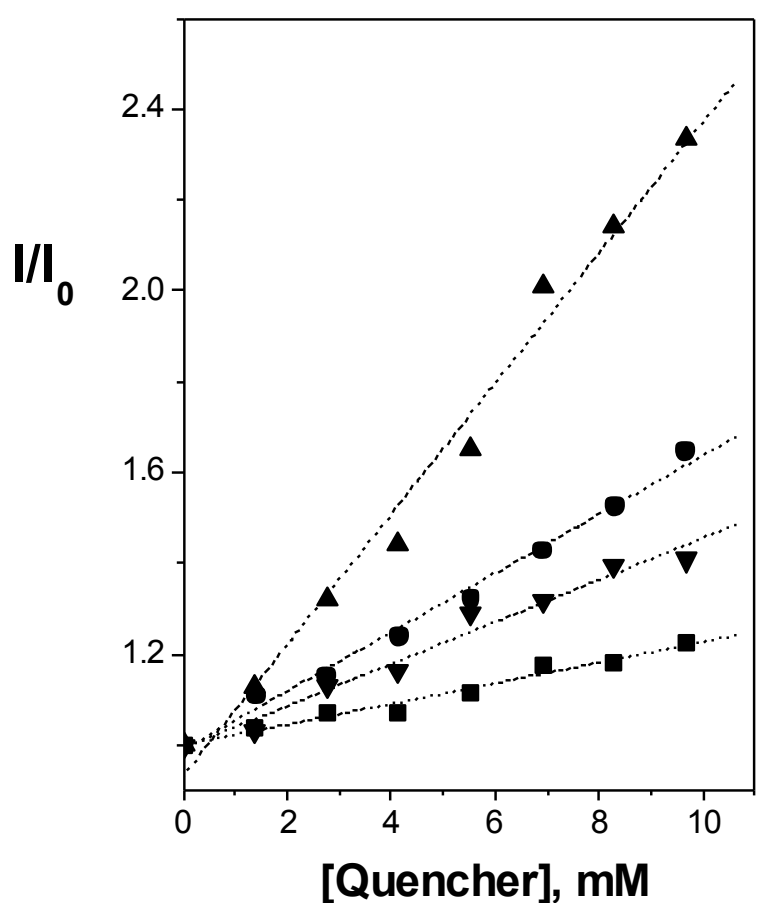

Table S4. Quenching parameters obtained from the Stern-Volmer depicted in Figure S3 for 9a12a $/ \mathrm{CH}_{2} \mathrm{Cl}_{2}$ dilute solutions at $25^{\circ} \mathrm{C}$ by using as quencher diacetyl.

\begin{tabular}{ccc}
\hline Dendrimer & $\mathbf{K}_{\mathbf{S V}}\left(\mathbf{M}^{\mathbf{- 1}}\right)$ & $\mathbf{k}_{\mathbf{q}}\left(\mathbf{1 0}^{\mathbf{9}} \mathbf{M}^{\mathbf{- 1}} \mathbf{s}^{\mathbf{- 1}}\right)$ \\
\hline 9a & $39.8 \pm 7.7$ & $9.0 \pm 1.8$ \\
10a & $17.1 \pm 1.6$ & $4.3 \pm 0.4$ \\
11a & $15.5 \pm 1.8$ & $5.2 \pm 0.6$ \\
12a & $15.7 \pm 3.8$ & $5.1 \pm 1.2$ \\
\hline
\end{tabular}




\section{Theoretical background}

Molecular Dynamics (MD) calculations were performed with Sybyl $8.0^{\mathrm{S} 9}$ and the Tripos Force Field. ${ }^{\mathrm{S} 10}$ Geometry for the carbene N-heterocyclic palladium core was obtained from the X-ray structure and the charges by the DFT (B3LYP/6-31g*) method. ${ }^{\mathrm{S} 11}$ Charges for dendrimeric substituents were obtained by MOPAC. ${ }^{\text {S12 }}$ A relative permittivity $\varepsilon=1$ was used. Non-bonded cutoff distances were set at $8 \AA$. Optimizations were carried out by the simplex algorithm, and the conjugate gradient was used as a termination method with gradients of $0.05 \mathrm{Kcal} / \mathrm{mol} \AA$ for the initial structures. ${ }^{\mathrm{S} 13, \mathrm{~S} 14}$ During the MD simulations the carbene N-heterocyclic palladium core was fixed as depicted in Figure S4, i.e., the Pd atom was located at the center of a coordinate system, the carbene N-heterocyclic groups were in the $\mathrm{xz}$ plane and the bromide atoms followed the $\mathrm{y}$ direction.

To study the conformational behavior for the $\mathrm{G} n$ dendrimers, $5 \mathrm{~ns}$ MD trajectories in the vacuo were performed on the trans-syn and trans-anti conformers depicted in Scheme 2 of the manuscript. Starting from $1 \mathrm{~K}$ the temperature was increased by $20 \mathrm{~K}$ intervals and the whole system equilibrated at each intermediate temperature for 400 fs up to reaching the temperature of $500 \mathrm{~K}$. Once at this temperature, an additional equilibration period of up to $10^{5} \mathrm{fs}$ was used. The whole heating/equilibration period was discharged from the analysis. From this point, the rest of the trajectory was simulated at $1 \mathrm{fs}$ integration time steps up to completing a time of $5 \mathrm{~ns}$. The velocities were rescaled at 10 fs intervals. Structures obtained from the analysis of MD trajectories in vacuo were saved every 200 fs yielding 25,000 images for subsequent analysis. The averages of any property obtained from the MD trajectories analysis were calculated by equally weighing each image. 
Figure S4. Coordinate system used for 9a-12a dendrimers in the theoretical MD simulations, exemplified for 10a.

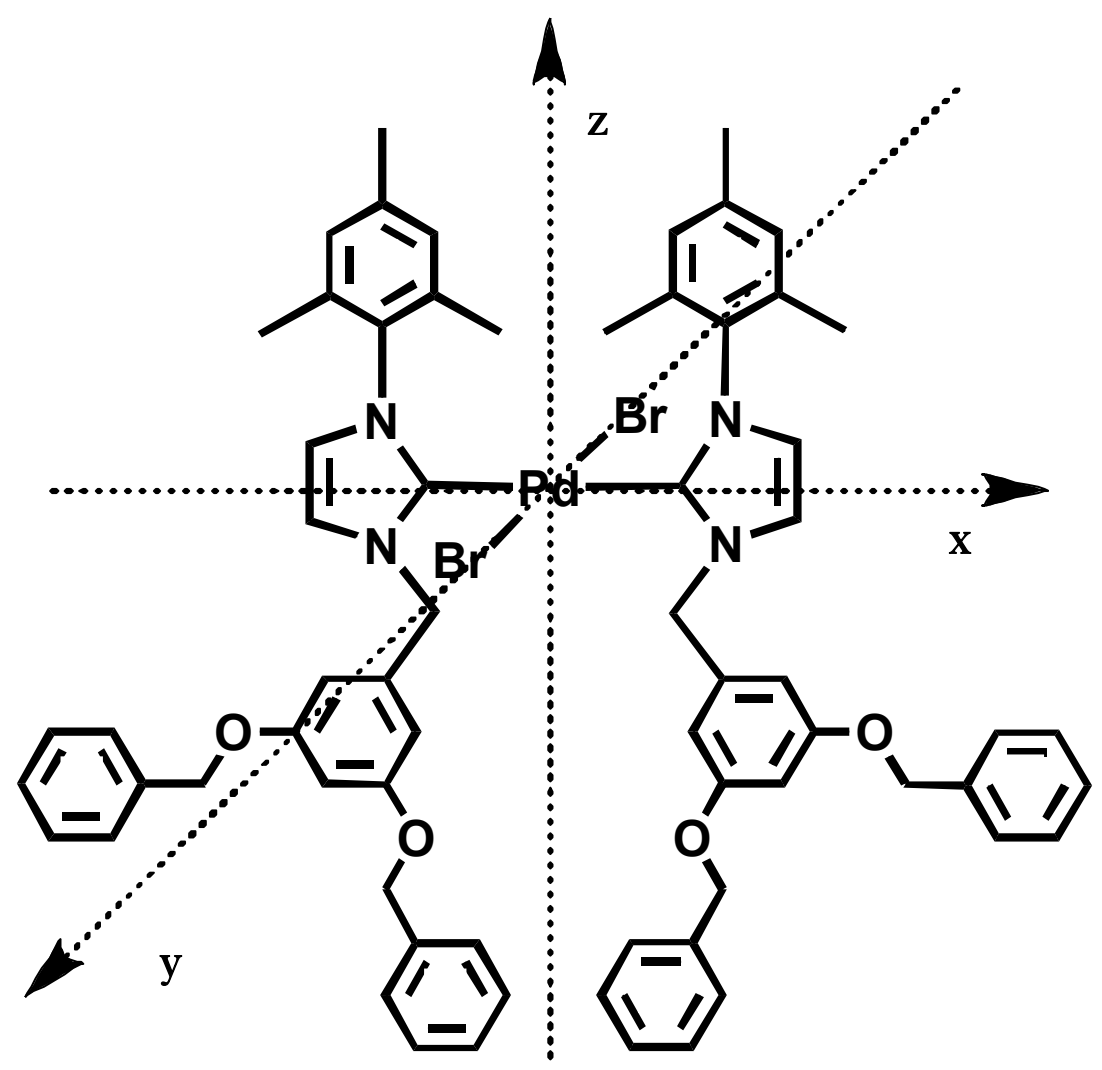


Figure S5. (Upper panel) Hydrodynamics volumes for 9a-12a in DMF (ם), acetone (•) (both from DOSY NMR experiments) obtained at $25{ }^{\circ} \mathrm{C}$. Van der Waals $(\nabla)$ and total volumes $(\square)$ obtained from van der Waals radii and from the values of the average of the root-mean-square radius of gyration respectively. The latter obtained from the analysis of the $5 \mathrm{~ns}$ MD trajectories at $300 \mathrm{~K}$ under the assumption of a 2:1 mixture of trans-syn and trans-anti rotamers. (Bottom panel) experimental and theoretical dendrimer densities obtained from the volumes of the upper panel.
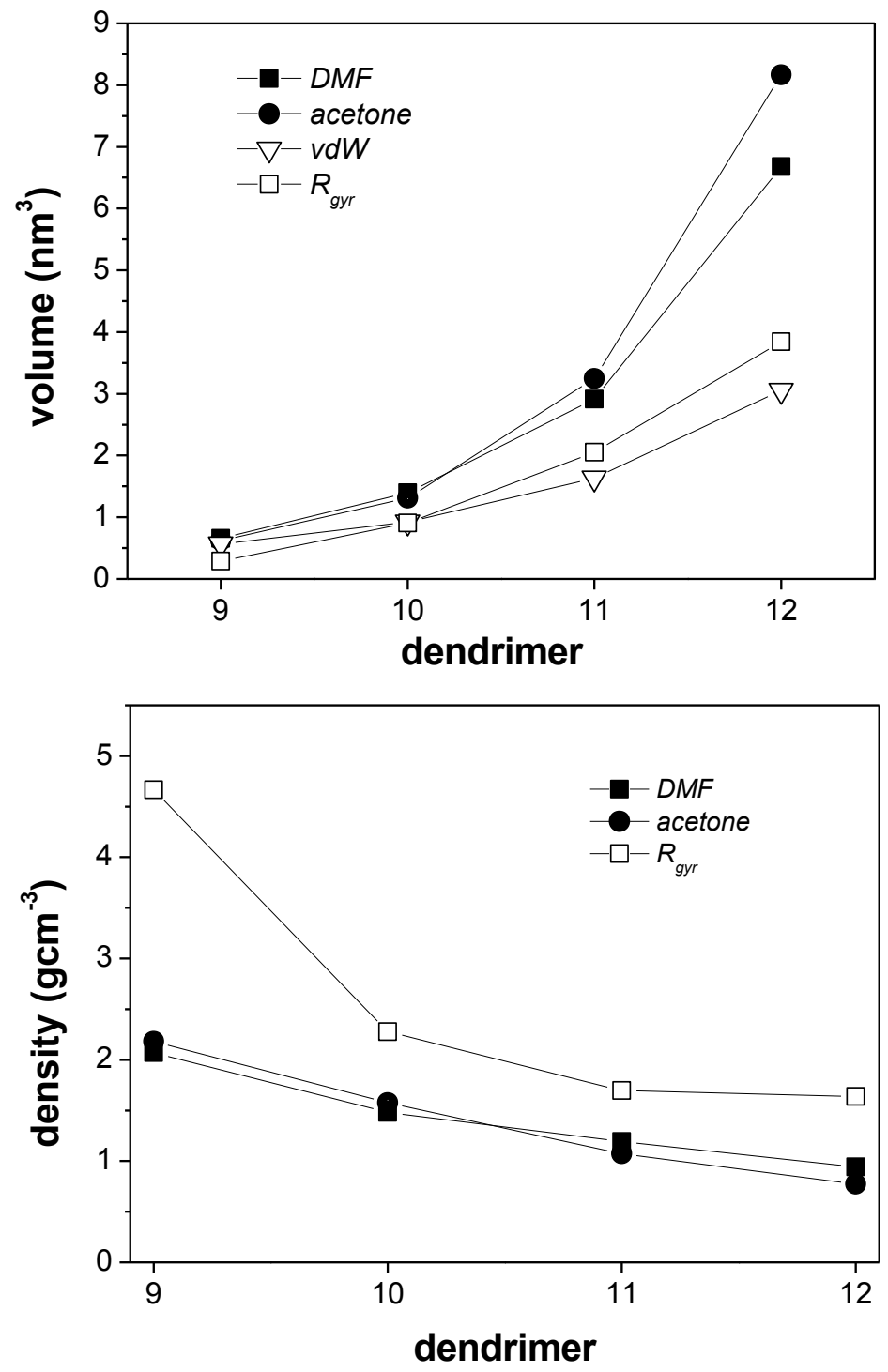
Figure S6. Density of the centers of mass of the phenyl ring from dendrons and mesityl groups (number of centers per $\AA^{3}$ ) from the analysis of the $5 \mathrm{~ns}$ MD trajectories at $300 \mathrm{~K}$ for the trans-syn and trans-anti rotamers of the four dendrimer generations (G0-G4) as a function of the $r$ polar coordinate, the Pd-core being located at the center of a coordinate system (see Figure S4).
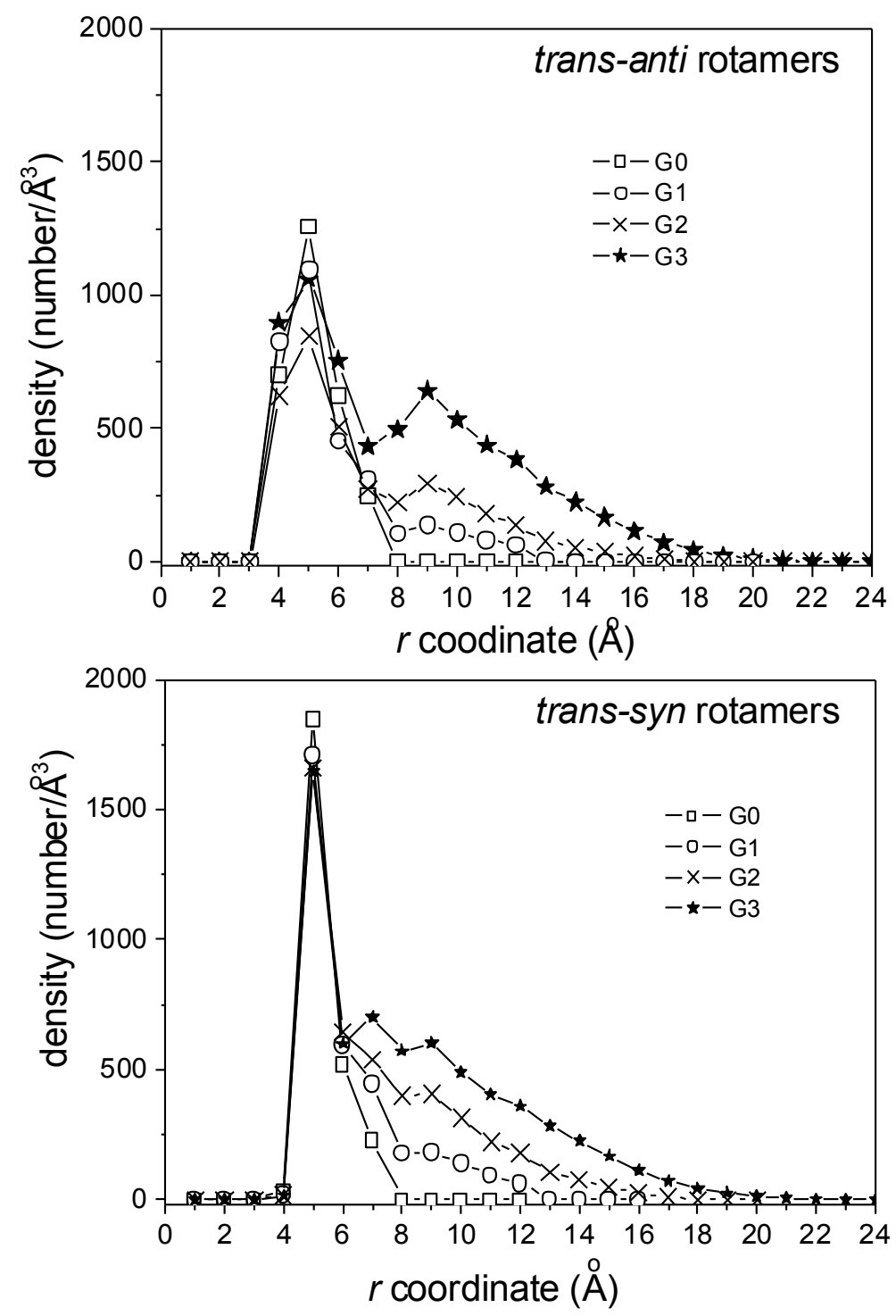


\section{References}

(S1) (a) Johnson, A. L., U. S. 3637731 1972. (b) Gardiner, M. G.; Herrmann, W. A.; Reisinger, C.-P.; Schwarz, J.; Spiegler, M. J. Organomet. Chem. 1999, 572, 239-247. (c) Liu, J.; Chen, J.; Zhao, J.; Zhao, Y.; Li, L.; Zhang, H. Synthesis 2003, 17, 2661-2666.

(S2) Drew, D.; Doyle, J. R. in Inorganic Syntheses, Vol. 13 (Ed. F. A. Cotton), McGraw-Hill Book Company, New York, 1972, pp. 47-52.

(S3) (a) Hawker, C. J.; Fréchet, J. M. J. J. Chem. Soc., Chem. Commun. 1990, 1010-1013. (b) Hawker C. J.; Fréchet, J. M. J. J. Am. Chem. Soc. 1990, 112, 7638-7647.

(S4) Perrin, D. D.: Armarego, W. L. F. in Purification of Laboratory Chemicals, 3rd ed., Pergamon Press, Oxford, 1988.

(S5) Sheldrick, G. M. SHELXL_97. Program for Crystal Structure Refinement; University of Göttingen, Göttingen (Germany), 1997.

(S6) Farrugia, L. J. WinGX System, J. Appl. Cryst., 1999, 32, 837.

(S7) O'Connor, D. V.; Ware,W.R.; André, J.C. J. Phys. Chem. 83, 1333 (1979).

(S8) Lakowicz, J. R. Principles of Fluorescence Spectroscopy, Springer, New York, 2006; p. 278.

(S9) Sybyl 8.0; Tripos Associates; St. Louis, Missouri, USA

(S10) Clark, M.; Cramer III, R. C.; Van Opdenbosch, N. J. Comp. Chem. 1989, 10, 982.

(S11) Frisch, M. J.; Trucks, G. W.; Schlegel, H. B.; Scuseria, G. E.; Robb, M. A.; Cheeseman, J. R.; Scalmani, G.; Barone, V.; Mennucci, B.; Petersson, G. A.; Nakatsuji, H.; Caricato, M.; Li, X.; Hratchian, H. P.; Izmaylov, A. F.; Bloino, J.; Zheng, G.; Sonnenberg, J. L.; Hada, M.; Ehara, M.; Toyota, K.; Fukuda, R.; Hasegawa, J.; Ishida, M.; Nakajima, T.; Honda, Y.; Kitao, O.; Nakai, H.; Vreven, T.; Montgomery, Jr., J. A.; Peralta, J. E.; Ogliaro, F.; Bearpark, M.; Heyd, J. J.; Brothers, E.; Kudin, K. N.; Staroverov, V. N.; Kobayashi, R.; Normand, J.; Raghavachari, K.; Rendell, A.; Burant, J. C.; Iyengar, S. S.; Tomasi, J.; Cossi, M.; Rega, N.; Millam, J. M.; Klene, M.; Knox, J. E.; Cross, J. B.; Bakken, V.; Adamo, C.; Jaramillo, J.; Gomperts, R.; Stratmann, R. E.; Yazyev, O.; Austin, A. J.; Cammi, R.; Pomelli, C.; Ochterski, J. W.; Martin, R. L.; Morokuma, K.; Zakrzewski, V. G.; Voth, G. A.; Salvador, P.; Dannenberg, J. J.; Dapprich, S.; Daniels, A. D.; Farkas, Ö.; Foresman, J. B.; Ortiz, J. V.; Cioslowski, J.; Fox, D. J. Gaussian 09, Revision A.1, ; Gaussian, Inc.,: Wallingford, CT, USA, 2009.

(S12) MOPAC (AM1), included in the Sybyl 6.9 package.

(S13) Brunel, Y. ; Faucher, H. ; Gagnaire, D. ; Rasat, A. Tetrahedron, 1975, 31, 1075.

(S14) Press, W. H.; Flannery, B. P.; Teukolski, S. A.; Vetterling, W. T. Numerical Recipes: The Art of Scientific Computing, Cambridge University Press, Cambridge, 1988; p. 312. 